SUPPORTING MATERIAL

\title{
An unusual radical Smiles rearrangement
}

Eric Bacqué ${ }^{1}$, Myriem El Qacemi ${ }^{2}$ and Samir Zard ${ }^{2} *$

${ }^{1}$ Sanofi-Aventis, 94400 Vitry sur Seine, France.

${ }^{2}$ Laboratoire de Synthèse Organique associé au CNRS

Ecole Polytechnique, 91128 Palaiseau, France

4. ${ }^{1} \mathrm{H}$ and ${ }^{13} \mathrm{C}$ spectra of compound $\mathbf{1 6}$

5. ${ }^{1} \mathrm{H}$ and ${ }^{13} \mathrm{C}$ spectra of compound $\mathbf{3 0}$ 


\section{Materials and Methods.}

Commercial solvents and reagents were used as received without further purification.

NMR spectra were recorded using a Bruker AMX400 operating at $400 \mathrm{MHz}$ for ${ }^{1} \mathrm{H}$ and 100 $\mathrm{MHz}$ for ${ }^{13} \mathrm{C}$. Chemical shifts are reported in parts per million (ppm). Coupling constants are in Hertz $(J \mathrm{~Hz})$; the following abbreviations are used for the description of signals: s (singlet), $\mathrm{d}$ (doublet), dd (double doublet), $\mathrm{t}$ (triplet), q (quadruplet), m (multiplet).

Mass spectra were recorded with a HP 5989B mass spectrometer using electron impact (EI) and electron spray ionization (ESI).

Analytical TLC was carried out on Merck silica gel plates using short wave (254 nm) UV light, $\mathrm{KMnO}_{4}$, anisaldehyde and vanilline staining to visualise components.

Infra-red spectra were recorded with a Perkin-Elmer 1600 Fourier Transform Spectrophotometer and are reported in terms of frequency of absorption $\left(\mathrm{cm}^{-1}\right)$.

Silica gel (Silice 60, A C.C 40-63, SDS) was used for flash column chromatography. Melting points were determined by Reichert microscope apparatus and were uncorrected.

\section{Representative procedures}

a. Preparation of the $N$-substituted arylamines.

\section{$\underline{\text { Method } 1}$}<smiles>CCOC(=O)C(C)(C)OC(=N)C(C)(C)C</smiles>

To a stirred solution of the aniline in cyclohexane $(0.5 \mathrm{~mol} / \mathrm{L})$ at r.t. under argon, were successively added tert-Butyl 2,2,2-trichloroacetimidate (2.5 eq.) and a few drops of $\mathrm{BF}_{3} \cdot \mathrm{OEt}_{2}$. The formation of a white precipitate was usually observed. The reaction was monitored by TLC and if needed, tert-Butyl 2,2,2-trichloroacetimidate and $\mathrm{BF}_{3}$. $\mathrm{OEt}_{2}$ were added again. When starting material was totally consumed, the cyclohexane was evaporated in vacuo and the resulting crude residue was purified by flash column chromatography.

\section{Method 2}<smiles>Nc1cccnc1Cl</smiles><smiles>CCOC(C)(C)CC</smiles>

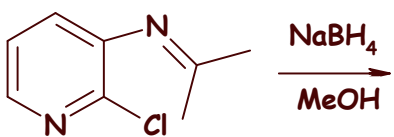<smiles>CC(C)Nc1cccnc1Cl</smiles>

A solution of the 3-amino-2-chloropyridine was heated at $120^{\circ} \mathrm{C}$ with 2,2 diethoxypropane (1.9 eq) for $20 \mathrm{~min}$. The excess of 2,2-diethoxypropane was then removed by evaporation in vacuo and the crude imine was dissolved in methanol $(0.3 \mathrm{~mol} / \mathrm{L})$ and stirred under argon. $\mathrm{NaBH}_{4}$ was then added in portionwise until all starting material was consumed. The reaction mixture was then quenched by adding water, extracted with dichloromethane, dried and concentrated under reduced pressure. The residue was purified by flash column chromatography. 
b. Preparation of the radical precursors :

\section{Method 3}<smiles>CCOS(=O)(=O)CC(=O)N(c1ccccc1Cl)C(C)(C)C(C)(C)Nc1ccccc1S(C)(=O)=O</smiles>

To a stirred solution of compound SM1 in dichloromethane or 1,2-dichloroethane $(0.1$ $\mathrm{mol} / \mathrm{L}$ ) at r.t. under argon, were successively added $N, N$-dimethylaniline (1.4 eq) and chloroacetyl chloride $(1.1 \mathrm{eq})$. The solution was then stirred at r.t. or refluxed until all starting material was totally consumed. The crude mixture was then poured in saturated aqueous $\mathrm{Na}_{2} \mathrm{CO}_{3}$, extracted with dichloromethane, dried and concentrated under reduced pressure. The residue was used with no further purification in the next reaction.

To a solution of the residue in acetone $(0.1 \mathrm{~mol} / \mathrm{L})$ under argon was added Potassium $O$ Ethyl xanthate $(2.5 \mathrm{eq})$ and the reaction mixture was stirred for $90 \mathrm{~min}$. at r.t.. After consumption of all starting material, acetone was evaporated and the resulting mixture was diluted with water, extracted with dichloromethane, dried and concentrated. The residue was purified by flash column chromatography.

c. Radical rearrangements :

\section{Method 4}

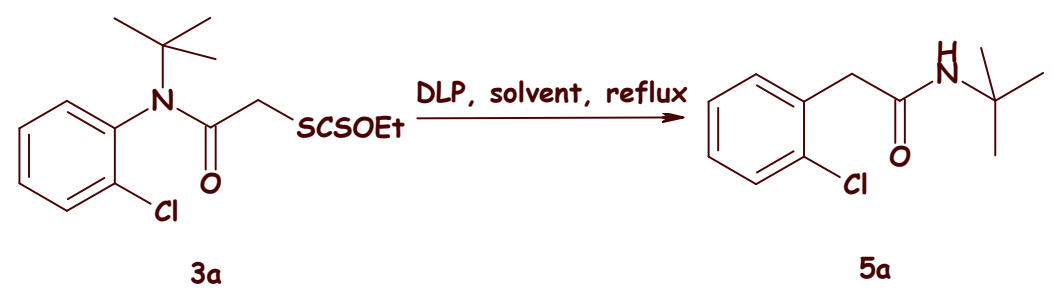

In a typical procedure, a solution of xanthate 3a in chlorobenzene, octane or di- $n$ butylether was refluxed for 15 min under argon. Lauroyl peroxide (DLP) was then added portionwise $(20 \mathrm{~mol} \%$ per 20 minutes) to the refluxing solution. The reaction was being regularly monitored for the disappearance of the starting material by thin-layer chromatography. The crude mixture was then cooled to room temperature, concentrated under reduced pressure and purified by flash column chromatography.

\section{Spectral Data}<smiles>CC(C)(C)Nc1cccnc1Cl</smiles>

tert-Butyl-(2-chloro-pyridin-3-yl)-amine was prepared according to the literature. ${ }^{1}$

\footnotetext{
${ }^{1}$ Morris, J.; Wishka, D. G. J. Org. Chem. 1995, 60, 2642-2644.
} 
<smiles>CC(C)(C)Nc1ccccc1Cl</smiles>

tert-Butyl-(2-chloro-phenyl)-amine was prepared according to Method 1 using 2chloroaniline $(0.45 \mathrm{~mL}, 4.28 \mathrm{mmol})$ and tert-Butyl 2,2,2-trichloroacetimidate $(2 \mathrm{~mL}, 11.2$ mmol). After completion of the reaction, the residue was purified by flash column chromatography (silica gel, petroleum ether-diethyl ether, 10:0 to 9:1) to give this compound in quantitative yield as a yellow oil. Its spectroscopic properties were identical to those described in the literature. ${ }^{2}$<smiles>CC(C)(C)Nc1ccc(Br)nc1Cl</smiles>

(6-Bromo-2-chloro-pyridin-3-yl)-tert-butyl-amine was prepared according to Method 1 using 6-bromo-2-chloro-3-aminopyridine ${ }^{3}(600 \mathrm{mg}, 2.90 \mathrm{mmol})$ and tert-Butyl 2,2,2trichloroacetimidate $(2 \mathrm{~mL}, 11.2 \mathrm{mmol}, 3.8 \mathrm{eq})$. After completion of the reaction, the residue was purified by flash column chromatography (silica gel, petroleum ether-diethyl ether, 10:0 to $93: 7$ ) to give this compound in $\mathbf{6 8 \%}$ yield as colorless crystals (m.p. $=97-100^{\circ} \mathrm{C}$, recrystallized with heptane/ethyl acetate). ${ }^{1} \mathrm{H} \mathrm{NMR}\left(\mathrm{CDCl}_{3}, 400 \mathrm{MHz}\right) \delta 7.19(\mathrm{~d}, 1 \mathrm{H}, \mathrm{CHar}$., $\mathrm{J}=8.5 \mathrm{~Hz}), 7.06$ (d, 1H, CHar., J=8.5Hz), 4.39 (bs, $1 \mathrm{H}, \mathrm{NH}), 1.39$ (s, 9H, $\left.\mathrm{CH}_{3}\right)$ ppm; ${ }^{13} \mathrm{C} \mathrm{NMR}$ $\left(\mathrm{CDCl}_{3}, 100 \mathrm{MHz}\right) \delta 139.1$ (Car.), 136.5 (Car.), 126.7 (CHar.), 123.6 (Car.), 122.4 (CHar.), $51.6(C), 29.5\left(\mathrm{CH}_{3}\right)$ ppm. IR $\left(\mathrm{CH}_{2} \mathrm{Cl}_{2}\right)$ 3416, 3061, 2978, 2935, 2874, 1577, 1489, 1461, $1413,1369,1326,1221,1144,1124,1071 \mathrm{~cm}^{-1}$. MS (CI+NH 3$) \mathrm{m} / \mathrm{z} 263,265,267\left(\mathrm{MH}^{+}\right)$.<smiles>CC(C)(C)Nc1c(Cl)cccc1Cl</smiles>

tert-Butyl-(2,6-dichloro-phenyl)-amine was obtained according to Method 1 using 2,6dichloroaniline $(1.017 \mathrm{~g}, 6.28 \mathrm{mmol})$ and tert-Butyl 2,2,2-trichloroacetimidate $(3.2 \mathrm{~mL}, 17.9$ mmol, $2.8 \mathrm{eq})$. After completion of the reaction, the residue was purified by flash column chromatography (silica gel, petroleum ether) to give this compound in $\mathbf{6 6 \%}$ yield as a colorless oil. ${ }^{1} \mathrm{H}$ NMR $\left(\mathrm{CDCl}_{3}, 400 \mathrm{MHz}\right) \delta 7.30$ (d, 1H, CHar., J=8.0Hz), 7.28 (d, 1H, CHar., $\mathrm{J}=8.0 \mathrm{~Hz}), 6.91(\mathrm{t}, 1 \mathrm{H}, \mathrm{CHar} ., \mathrm{J}=8.0 \mathrm{~Hz}), 3.50(\mathrm{bs}, 1 \mathrm{H}, \mathrm{NH}), 1.33\left(\mathrm{~s}, 9 \mathrm{H}, \mathrm{CH}_{3}\right) \mathrm{ppm} ;{ }^{13} \mathrm{C} \mathrm{NMR}$ $\left(\mathrm{CDCl}_{3}, 100 \mathrm{MHz}\right) \delta 141.9$ (Car.), 132.6 (Car.), 128.7 (CHar.), 124.2 (CHar.), 56.6 (C), 31.0 $\left(\mathrm{CH}_{3}\right) \mathrm{ppm}$. IR (neat) $3371,2968,1563,1441,1417,1364,1220,1188,795,761 \mathrm{~cm}^{-1} . \mathrm{MS}$ $\left(\mathrm{CI}+\mathrm{NH}_{3}\right) \mathrm{m} / \mathrm{z} 218,220,222\left(\mathrm{MH}^{+}\right)$.

\footnotetext{
${ }^{2}$ The yields we obtained were better than what has been done previously: Gage, J. R.; Wagner, J. M. J. Org. Chem. 1995, 60, 2613-2614.

${ }^{3}$ Prepared by reaction of N-Bromosuccinimide (1.2 eq) with 3-amino-2-chloropyridine in dimethylformamide $(0.4 \mathrm{~mol} / \mathrm{L})$. After basic work up, the resulting crude residue was purified by flash column chromatography (silica gel, petroleum ether-diethyl ether, 10:0 to 9:1) to give the compound in $\mathbf{7 5 \%}$ yield as orange crystals. (m.p $=118-121{ }^{\circ} \mathrm{C}$, recrystallized with heptane/ethyl acetate).$^{1} \mathrm{H}$ NMR $\left(\mathrm{CDCl}_{3}, 400 \mathrm{MHz}\right) \delta 7.18(\mathrm{~d}, 1 \mathrm{H}, \mathrm{CHar}$., $\mathrm{J}=8.2 \mathrm{~Hz}), 6.95$ (d, $1 \mathrm{H}, \mathrm{CHar} ., \mathrm{J}=8.2 \mathrm{~Hz}), 4.18$ (bs, $1 \mathrm{H}, \mathrm{NH}) \mathrm{ppm} ;{ }^{13} \mathrm{C} \mathrm{NMR}\left(\mathrm{CDCl}_{3}, 100 \mathrm{MHz}\right) \delta 139.3$ (Car.), 135.2 (Car.), 127.4 (CHar.), 126.0 (Car.), 125.0 (CHar.) ppm. IR $\left(\mathrm{CH}_{2} \mathrm{Cl}_{2}\right) 3489,3395,1618,1559,1444,1434$, $1375,1300,1251,1123,1106,920 \mathrm{~cm}^{-1}$. MS $\left(\mathrm{CI}+\mathrm{NH}_{3}\right) \mathrm{m} / \mathrm{z} 207,209,211\left(\mathrm{MH}^{+}\right)$.
} 
<smiles>Cc1ccccc1NC(C)(C)C</smiles>

tert-Butyl-o-tolyl-amine was obtained according to Method 1 using $o$-toluidine $(0.57 \mathrm{~mL}$, $5.34 \mathrm{mmol})$ and tert-Butyl 2,2,2-trichloroacetimidate $(2.5 \mathrm{~mL}, 13.9 \mathrm{mmol})$. After completion of the reaction, the residue was purified by flash column chromatography (silica gel, petroleum ether-diethyl ether, 10:0 to $97: 3$ ) to give this compound in $\mathbf{8 6 \%}$ yield as a colorless oil. Its spectroscopic properties were identical to those in the literature. ${ }^{2}{ }^{1} \mathrm{H} \mathrm{NMR}\left(\mathrm{CDCl}_{3}\right.$, $400 \mathrm{MHz}) \delta 7.13$ (dd, 2H, CHar., J=7.3Hz, J=8.1Hz), 6.96 (d, 1H, CHar., J=8.1Hz), 6.71 (d, 1H, CHar., J=7.3Hz), 3.49 (bs, $1 \mathrm{H}, \mathrm{NH}), 2.18$ (s, 3H, $\left.\mathrm{CH}_{3}\right), 1.45$ (s, 9H, $\left.\mathrm{CH}_{3}\right)$ ppm; ${ }^{13} \mathrm{C} \mathrm{NMR}$ $\left(\mathrm{CDCl}_{3}, 100 \mathrm{MHz}\right) \delta 145.0$ (Car.), 130.5 (CHar.), 126.7 (CHar.), 123.6 (Car.), 117.1 (CHar.), 114.2 (CHar.), $51.3(C), 30.2\left(\mathrm{CH}_{3}\right), 18.2\left(\mathrm{CH}_{3}\right)$ ppm. IR (neat) 3438, 2968, 2868, 1606, $1586,1514,1481,1443,1364,1318,1264,1227,1054,744 \mathrm{~cm}^{-1}$.<smiles>CC(C)(C)Nc1ccccc1C(F)(F)F</smiles>

tert-Butyl-(2-trifluoromethyl-phenyl)-amine was obtained according to Method 1 using $O$ trifluorométhylaniline $(0.68 \mathrm{~mL}, 5.41 \mathrm{mmol})$ and tert-Butyl 2,2,2-trichloroacetimidate $(2.5$ $\mathrm{mL}, 13.9 \mathrm{mmol}, 2.6 \mathrm{eq})$. After completion of the reaction, the residue was purified by flash column chromatography (silica gel, petroleum ether-diethyl ether, 10:0 to 9:1) to give this compound in $\mathbf{9 8 \%}$ yield as a colorless oil. ${ }^{1} \mathrm{H} \mathrm{NMR}\left(\mathrm{CDCl}_{3}, 400 \mathrm{MHz}\right) \delta 7.43(\mathrm{~d}, 1 \mathrm{H}, \mathrm{CHar}$., $\mathrm{J}=7.9 \mathrm{~Hz}$ ), 7.32 (t, 1H, CHar., $\mathrm{J}=7.8 \mathrm{~Hz}), 7.02$ (d, 1H, CHar., J=8.4Hz), 6.70 (t, 1H, CHar., $\mathrm{J}=7.6 \mathrm{~Hz}), 4.29$ (bs, $1 \mathrm{H}, \mathrm{NH}), 1.42\left(\mathrm{~s}, 9 \mathrm{H}, \mathrm{CH}_{3}\right) \mathrm{ppm} ;{ }^{13} \mathrm{C} \mathrm{NMR}\left(\mathrm{CDCl}_{3}, 100 \mathrm{MHz}\right) \delta 145.2(\mathrm{q}$, Car., J=1.4Hz), 132.4 (q, CHar., J=0.9Hz), 126.9 (q, CHar., J=5.7Hz), 125.4 (q, $C \mathrm{~F}_{3}$, $\mathrm{J}=272.3 \mathrm{~Hz}$ ), 115.9 (s, CHar.), 115.6 (s, CHar.), 115.1 (q, Car., J=28.4Hz), 51.7 (s, C), 30.1 (s, $\mathrm{CH}_{3}$ ) ppm. IR (neat) 3474, 2979, 1616, 1589, 1526, 1482, 1331, 1289, 1214, 1166, 1100, $1036,752 \mathrm{~cm}^{-1}$. MS $\left(\mathrm{CI}+\mathrm{NH}_{3}\right) \mathrm{m} / \mathrm{z} 218\left(\mathrm{MH}^{+}\right)$.<smiles>CC(C)Nc1cccnc1Cl</smiles>

(2-Chloro-pyridin-3-yl)-isopropyl-amine was obtained according to Method 2, by heating first a solution of 3-amino-2-chloropyridine $(404 \mathrm{mg}, 3.21 \mathrm{mmol})$ at $120^{\circ} \mathrm{C}$ with $2,2-$ diethoxypropane $(1.02 \mathrm{~mL}, 6.32 \mathrm{mmol}, 2 \mathrm{eq})$ for $20 \mathrm{~min}$. The excess of 2,2-diethoxypropane was then removed by evaporation in vacuo and the crude imine was dissolved in methanol (10 $\mathrm{mL}$ ) and stirred under argon. $\mathrm{NaBH}_{4}(715 \mathrm{mg}, 18.9 \mathrm{mmol}, 5.9 \mathrm{eq})$ was then added portionwise until all starting material was consumed. After work up, the residue was purified by flash column chromatography (silica gel, petroleum ether-diethyl ether, 10:0 to 9:1) to give this compound in $91 \%$ yield over 2 steps as a colorless oil. ${ }^{1} \mathrm{H} \mathrm{NMR}\left(\mathrm{CDCl}_{3}, 400 \mathrm{MHz}\right) \delta 7.66$ (dd, 1H, CHar., J=1.4Hz, J=4.6Hz), 7.06 (dd, 1H, CHar., J=4.6Hz, J=8.0Hz), 6.87 (dd, 1H, CHar., J=1.4Hz, J=8.0Hz), 4.18 (bs, $1 \mathrm{H}, \mathrm{NH}), 3.60$ (m, 1H, $\mathrm{NCH}), 1.25$ (bd, 6H, $\mathrm{CH}_{2}$, $\mathrm{J}=6.3 \mathrm{~Hz}) \mathrm{ppm} ;{ }^{13} \mathrm{C} \mathrm{NMR}\left(\mathrm{CDCl}_{3}, 100 \mathrm{MHz}\right) \delta 140.1$ (Car.), 137.1 (Car.), 135.9 (CHar.), 123.4 (CHar.), 117.7 (CHar.), $44.0(\mathrm{CHN}), 22.7\left(\mathrm{CH}_{3}\right) \mathrm{ppm} . \mathrm{IR}$ (neat) 3415, 2970, 2932, 
$1584,1561,1484,1456,1384,1329,1212,1172,1055,788 \mathrm{~cm}^{-1} . \mathrm{MS}\left(\mathrm{CI}+\mathrm{NH}_{3}\right) \mathrm{m} / \mathrm{z} \mathrm{171}, 173$ $\left(\mathrm{MH}^{+}\right)$.<smiles>CCOC(=S)SCC(=O)N(c1cccnc1Cl)C(C)(C)C</smiles>

Dithiocarbonic acid \{[tert-butyl-(2-chloro-pyridin-3-yl)-carbamoyl]-methyl $\}$ ester ethyl ester 3a was prepared according to Method 3: The first reaction was carried out using a solution of the corresponding aminopyridine $(403 \mathrm{mg}, 2.20 \mathrm{mmol})$ in 1,2-dichloroethane $(20 \mathrm{~mL}), \mathrm{N}, \mathrm{N}$ dimethylaniline $(1 \mathrm{~mL}, 7.89 \mathrm{mmol}, 3.6 \mathrm{eq})$ and chloroacetyl chloride $(0.50 \mathrm{~mL}, 6.27 \mathrm{mmol}$, $2.8 \mathrm{eq})$. The solution was refluxed until starting material was totally consumed. After work up, the crude residue was treated with Potassium $O$-Ethyl xanthate $(870 \mathrm{mg}, 5.44 \mathrm{mmol}, 2.5$ eq) in acetone $(20 \mathrm{~mL})$ to afford, after work up and purification by flash column chromatography (silica gel, petroleum ether-diethyl ether, 10:0 to 7:3), 3a in quantitative yield over 2 steps as a yellow oil. ${ }^{1} \mathrm{H} \mathrm{NMR}\left(\mathrm{CDCl}_{3}, 400 \mathrm{MHz}\right) \delta 8.45(\mathrm{dd}, 1 \mathrm{H}, \mathrm{CHar}$, $\mathrm{J}=1.8 \mathrm{~Hz}, \mathrm{~J}=4.7 \mathrm{~Hz}$ ), 7.76 (dd, 1H, CHar., $\mathrm{J}=1.8 \mathrm{~Hz}, \mathrm{~J}=7.8 \mathrm{~Hz}$ ), 7.39 (dd, $1 \mathrm{H}, \mathrm{CHar} ., \mathrm{J}=4.7 \mathrm{~Hz}$, $\mathrm{J}=7.8 \mathrm{~Hz}), 4.57$ and $4.55\left(2 \mathrm{xq}, 2 \mathrm{H}, \mathrm{OCH}_{2}, \mathrm{~J}=7.1 \mathrm{~Hz}\right), 3.56\left(\mathrm{~d}, 1 \mathrm{H}, \mathrm{CH}_{2}, \mathrm{~J}=16.0 \mathrm{~Hz}\right), 3.53(\mathrm{~d}$, $\left.1 \mathrm{H}, \mathrm{CH}_{2}, \mathrm{~J}=16.0 \mathrm{~Hz}\right), 1.42\left(\mathrm{~s}, 9 \mathrm{H}, \mathrm{CH}_{3}\right), 1.36\left(\mathrm{t}, 3 \mathrm{H}, \mathrm{CH}_{3}, \mathrm{~J}=7.1 \mathrm{~Hz}\right) \mathrm{ppm} ;{ }^{13} \mathrm{C} \mathrm{NMR}\left(\mathrm{CDCl}_{3}\right.$, $100 \mathrm{MHz}$ ) $\delta 214.0$ (CS), 165.4 (CO), 152.1 (Car.), 149.6 (CHar.), 140.4 (CHar.), 136.2 (Car.), 123.2 (CHar.), $70.6\left(\mathrm{OCH}_{2}\right), 61.0(\mathrm{C}), 42.0\left(\mathrm{CH}_{2}\right), 29.0\left(\mathrm{CH}_{3}\right), 13.8\left(\mathrm{CH}_{3}\right)$ ppm. IR (neat) $2979,2935,1674,1558,1447,1402,1363,1344,1231,1190,1112,1050,757,735 \mathrm{~cm}^{-1}$. MS $\left(\mathrm{CI}+\mathrm{NH}_{3}\right) \mathrm{m} / \mathrm{z} 347,349\left(\mathrm{MH}^{+}\right)$.<smiles>CCOC(=S)SCC(=O)N(c1ccc(Br)nc1Cl)C(C)(C)C</smiles>

Dithiocarbonic acid \{[(6-bromo-2-chloro-pyridin-3-yl)-tert-butyl-carbamoyl]-methyl $\}$ ester ethyl ester 3b was prepared according to Method 3: The first reaction was carried out using a solution of the corresponding aminopyridine $(281 \mathrm{mg}, 1.06 \mathrm{mmol})$ in 1,2-dichloroethane (20 $\mathrm{mL}), N, N$-dimethylaniline $(0.50 \mathrm{~mL}, 3.94 \mathrm{mmol}, 3.7 \mathrm{eq})$ and chloroacetyl chloride $(0.52 \mathrm{~mL}$, $6.53 \mathrm{mmol}, 6.1 \mathrm{eq})$. The solution was refluxed until starting material was totally consumed. After work up, the crude residue was treated with Potassium $O$-Ethyl xanthate $(500 \mathrm{mg}, 1.25$ mmol, $1.2 \mathrm{eq})$ in acetone $(10 \mathrm{~mL})$ to afford, after work up and purification by flash column chromatography (silica gel, petroleum ether-diethyl ether, 10:0 to 8:2) followed by recrystallization in heptane, $\mathbf{5 4 \%}$ yield over 2 steps of $\mathbf{3 b}$ as yellow crystals. (m.p. $=118$ $120^{\circ} \mathrm{C}$, recrystallized with heptane). ${ }^{1} \mathrm{H}$ NMR $\left(\mathrm{CDCl}_{3}, 400 \mathrm{MHz}\right) \delta 7.62(\mathrm{~d}, 1 \mathrm{H}, \mathrm{CHar}$., $\mathrm{J}=8.1 \mathrm{~Hz}), 7.56(\mathrm{~d}, 1 \mathrm{H}, \mathrm{CHar} ., \mathrm{J}=8.1 \mathrm{~Hz}), 4.59$ and $4.57\left(2 \mathrm{xq}, 2 \mathrm{H}, \mathrm{OCH}_{2}, \mathrm{~J}=7.1 \mathrm{~Hz}\right), 3.60(\mathrm{~d}$, $\left.1 \mathrm{H}, \mathrm{CH}_{2}, \mathrm{~J}=15.9 \mathrm{~Hz}\right), 3.53\left(\mathrm{~d}, 1 \mathrm{H}, \mathrm{CH}_{2}, \mathrm{~J}=15.9 \mathrm{~Hz}\right), 1.42\left(\mathrm{~s}, 9 \mathrm{H}, \mathrm{CH}_{3}\right), 1.38\left(\mathrm{t}, 3 \mathrm{H}, \mathrm{CH}_{3}\right.$, $\mathrm{J}=7.1 \mathrm{~Hz})$ ppm; ${ }^{13} \mathrm{C} \mathrm{NMR}\left(\mathrm{CDCl}_{3}, 100 \mathrm{MHz}\right) \delta 214.0$ (CS), 165.3 (CO), 151.4 (Car.), 142.1 (CHar.), 140.2 (Car.), 135.6 (Car.), 127.8 (CHar.), $70.8\left(\mathrm{OCH}_{2}\right), 61.2(C), 41.8\left(\mathrm{CH}_{2}\right), 29.0$ $\left(\mathrm{CH}_{3}\right), 13.8\left(\mathrm{CH}_{3}\right)$ ppm. IR $\left(\mathrm{CH}_{2} \mathrm{Cl}_{2}\right) 3061,2983,2938,1673,1537,1427,1418,1366,1335$, 1236, 1188, 1123, 1095, 1051, $956 \mathrm{~cm}^{-1}$. MS (CI+NH$) \mathrm{m} / \mathrm{z} 426,428,430\left(\mathrm{MH}^{+}\right)$. 
<smiles>CCOC(=S)SCC(=O)N(C)c1cccnc1Cl</smiles>

Dithiocarbonic acid $\{[(2$-chloro-pyridin-3-yl)-methyl-carbamoyl]-methyl $\}$ ester ethyl ester 3c was prepared according to Method 3: The first reaction was carried out using a solution of (2Chloro-pyridin-3-yl)-methyl-amine ${ }^{4}(490 \mathrm{mg}, 3.43 \mathrm{mmol})$ in dichloromethane $(20 \mathrm{~mL})$ and chloroacetyl chloride $(0.55 \mathrm{~mL}, 6.90 \mathrm{mmol}, 2 \mathrm{eq})$. The solution was stirred at r.t. until starting material was totally consumed. After work up, the crude residue was treated with Potassium $O$-Ethyl xanthate $(856 \mathrm{mg}, 5.35 \mathrm{mmol}, 1.6 \mathrm{eq})$ in acetone $(35 \mathrm{~mL})$ to afford, after work up and purification by flash column chromatography (silica gel, petroleum ether-diethyl ether, 10:0 to $3: 7), 3 \mathrm{c}$ in $\mathbf{9 7 \%}$ yield over 2 steps as a colorless oil. ${ }^{1} \mathrm{H}$ NMR $\left(\mathrm{CDCl}_{3}, 400 \mathrm{MHz}\right) \delta 8.43(\mathrm{~d}$, $1 \mathrm{H}, \mathrm{CHar} ., \mathrm{J}=4.7 \mathrm{~Hz}$ ), 7.82 (d, 1H, CHar., J=7.8Hz), 7.40 (dd, $1 \mathrm{H}$ CHar., J=4.7Hz, J=7.8Hz), 4.53 (q, 2H, OCH $\left.H_{2}, \mathrm{~J}=7.1 \mathrm{~Hz}\right), 3.75\left(\mathrm{~d}, 1 \mathrm{H}, \mathrm{CH}_{2}, \mathrm{~J}=15.7 \mathrm{~Hz}\right), 3.64\left(\mathrm{~d}, 1 \mathrm{H}, \mathrm{CH}_{2}, \mathrm{~J}=15.7 \mathrm{~Hz}\right)$, $3.22\left(\mathrm{~s}, 3 \mathrm{H}, \mathrm{CH}_{3}\right), 1.33\left(\mathrm{t}, 3 \mathrm{H}, \mathrm{CH}_{3}, \mathrm{~J}=7.1 \mathrm{~Hz}\right) \mathrm{ppm} ;{ }^{13} \mathrm{C} \mathrm{NMR}\left(\mathrm{CDCl}_{3}, 100 \mathrm{MHz}\right) \delta 213.2$ (CS), 166.2 (CO), 150.3 (Car.), 149.7 (CHar.), 138.9 (CHar.), 137.0 (Car.), 123.9 (CHar.), $70.6\left(\mathrm{OCH}_{2}\right), 39.2\left(\mathrm{CH}_{2}\right), 36.4\left(\mathrm{CH}_{3}\right), 13.7\left(\mathrm{CH}_{3}\right) \mathrm{ppm}$. IR (neat) 2980, 2936, 1674, 1560, $1455,1407,1361,1224,1145,1112,1048,750 \mathrm{~cm}^{-1}$. MS $\left(\mathrm{CI}+\mathrm{NH}_{3}\right) \mathrm{m} / \mathrm{z} \mathrm{305,} 307\left(\mathrm{MH}^{+}\right)$.<smiles>CCOC(=S)SCC(=O)N(c1cccnc1Cl)C1CCCCC1</smiles>

Dithiocarbonic acid $\{[(2-c h l o r o-p y r i d i n-3-y l)-c y c l o h e x y l-c a r b a m o y l]-m e t h y l\}$ ester ethyl ester 3e was prepared according to Method 2 and Method 3 : by heating first a solution of 3amino-2-chloropyridine $(550 \mathrm{mg}, 4.36 \mathrm{mmol})$ at $120^{\circ} \mathrm{C}$ with 1,1 -diethoxycyclohexane ${ }^{5}(9.65$ $\mathrm{mmol}, 2.2 \mathrm{eq})$ for $40 \mathrm{~min}$. The excess of 1,1-diethoxycyclohexane was then removed by evaporation in vacuo and the crude imine was dissolved in methanol $(4 \mathrm{~mL})$ and stirred under argon. $\mathrm{NaBH}_{4}(1.160 \mathrm{~g}, 30.7 \mathrm{mmol}, 7.0 \mathrm{eq})$ was then added portionwise until all starting material was consumed. After work up, the residue was dissolved in 1,2-dichloroethane (20 $\mathrm{mL})$, and treated with $N, N$-dimethylaniline $(0.89 \mathrm{~mL}, 7.02 \mathrm{mmol}, 1.6 \mathrm{eq})$ and chloroacetyl chloride $(0.42 \mathrm{~mL}, 5.27 \mathrm{mmol}, 1.2 \mathrm{eq})$. The solution was stirred at r.t. until starting material was totally consumed. After work up, the crude residue was treated with Potassium $O$-Ethyl xanthate $(1.272 \mathrm{~g}, 7.95 \mathrm{mmol}, 1.8 \mathrm{eq})$ in acetone $(30 \mathrm{~mL})$ to give, after work up and purification by flash column chromatography (silica gel, petroleum ether-diethyl ether, 10:0 to $1: 1)$ a $\mathbf{7 3 \%}$ yield of $\mathbf{3 e}$ over 4 steps as a yellow oil. ${ }^{1} \mathrm{H}$ NMR $\left(\mathrm{CDCl}_{3}, 400 \mathrm{MHz}\right) \delta 8.46(\mathrm{dd}$, 1H, CHar., J=1.7Hz, J=4.7Hz), 7.77 (dd, 1H, CHar., J=1.7Hz, J=7.8Hz), 7.40 (dd, 1H, CHar., $\mathrm{J}=4.7 \mathrm{~Hz}, \mathrm{~J}=7.8 \mathrm{~Hz}), 4.60-4.52(\mathrm{~m}, 1 \mathrm{H}, \mathrm{NCH}), 4.55\left(\mathrm{~m}, 2 \mathrm{H}, \mathrm{OCH}_{2}, \mathrm{~J}=7.1 \mathrm{~Hz}\right), 3.67(\mathrm{~d}, 1 \mathrm{H}$, $\left.\mathrm{CH}_{2}, \mathrm{~J}=15.9 \mathrm{~Hz}\right), 3.63\left(\mathrm{~d}, 1 \mathrm{H}, \mathrm{CH}_{2}, \mathrm{~J}=15.9 \mathrm{~Hz}\right), 2.03(\mathrm{~d}, 1 \mathrm{H}, \mathrm{CH} 2, \mathrm{~J}=11.7 \mathrm{~Hz}), 1.95(\mathrm{~d}, 1 \mathrm{H}$, $\left.\mathrm{CH}_{2}, \mathrm{~J}=11.7 \mathrm{~Hz}\right), 1.73(\mathrm{~m}, 2 \mathrm{H}), 1.59\left(\mathrm{~d}, 1 \mathrm{H}, \mathrm{CH}_{2}, \mathrm{~J}=13.2 \mathrm{~Hz}\right), 1.41-1.32\left(\mathrm{~m}, 2 \mathrm{H}, \mathrm{CH}_{2}\right), 1.36(\mathrm{t}$, $\left.3 \mathrm{H}, \mathrm{CH}_{3}, \mathrm{~J}=7.1 \mathrm{~Hz}\right), 1.25-1.15\left(\mathrm{~m}, 1 \mathrm{H}, \mathrm{CH}_{2}\right), 0.99-0.81\left(\mathrm{~m}, 2 \mathrm{H}, \mathrm{CH}_{2}\right) \mathrm{ppm} ;{ }^{13} \mathrm{C} \mathrm{NMR}$ $\left(\mathrm{CDCl}_{3}, 100 \mathrm{MHz}\right) \delta 213.8$ (CS), 165.6 (CO), 152.2 (Car.), 149.6 (CHar.), 140.7 (CHar.),

\footnotetext{
${ }^{4}$ Zakrzewski, P.; Gowan, M.; Trimble, L. A.; Lau, C. K. Synthesis, 1999, 11, 1893-1902.

${ }^{5}$ Gopinath, R.; Haque, S. J.; Patel, B. K. J. Org. Chem. 2002, 67, 5842-5845.
} 
133.7 (Car.), 123.2 (CHar.), $70.7\left(\mathrm{OCH}_{2}\right), 57.2(\mathrm{NCH}), 40.0\left(\mathrm{CH}_{2}\right), 32.2\left(\mathrm{CH}_{2}\right), 29.9\left(\mathrm{CH}_{2}\right)$, $25.7\left(\mathrm{CH}_{2}\right), 25.4\left(\mathrm{CH}_{2}\right), 13.8\left(\mathrm{CH}_{3}\right) \mathrm{ppm}$. IR (neat) 2980, 2933, 1681, 1558, 1454, 1404, 1384, $1360,1323,1233,1149,1112,1049,753,731 \mathrm{~cm}^{-1}$. MS $\left(\mathrm{CI}+\mathrm{NH}_{3}\right) \mathrm{m} / \mathrm{z} 372,374\left(\mathrm{MH}^{+}\right)$.<smiles>CCOC(=S)SCC(=O)N(c1cccnc1Cl)C(C)C</smiles>

Dithiocarbonic acid $\{[(2-c h l o r o-p y r i d i n-3-y l)$-isopropyl-carbamoyl $]$-methyl $\}$ ester ethyl ester 3d was prepared according to Method 3: The first reaction was carried out using a solution of the corresponding aminopyridine $(400 \mathrm{mg}, 2.35 \mathrm{mmol})$ in dichloromethane $(23 \mathrm{~mL}), N, N$ dimethylaniline $(0.90 \mathrm{~mL}, 7.10 \mathrm{mmol}, 3 \mathrm{eq})$ and chloroacetyl chloride $(0.47 \mathrm{~mL}, 5.90 \mathrm{mmol}$, $2.5 \mathrm{eq})$. The solution was refluxed until starting material was totally consumed. After work up, the crude residue was treated with Potassium $O$-Ethyl xanthate $(720 \mathrm{mg}, 4.5 \mathrm{mmol}, 1.9$ eq) in acetone $(23 \mathrm{~mL})$ to afford, after work up and purification by flash column chromatography (silica gel, petroleum ether-diethyl ether, 10:0 to 1:1), 3d in quantitative yield over 2 steps as a yellow oil. ${ }^{1} \mathrm{H} \mathrm{NMR}\left(\mathrm{CDCl}_{3}, 400 \mathrm{MHz}\right) \delta 8.47$ (dd, $1 \mathrm{H}, \mathrm{CHar}$., $\mathrm{J}=1.7 \mathrm{~Hz}, \mathrm{~J}=4.7 \mathrm{~Hz}$ ), 7.77 (dd, 1H, CHar., $\mathrm{J}=1.7 \mathrm{~Hz}, \mathrm{~J}=7.8 \mathrm{~Hz}$ ), 7.41 (dd, 1H, CHar., $\mathrm{J}=4.7 \mathrm{~Hz}$, $\mathrm{J}=7.8 \mathrm{~Hz}), 4.90(\mathrm{~m}, 1 \mathrm{H}, \mathrm{NCH}), 4.54\left(\mathrm{dq}, 2 \mathrm{H}, \mathrm{OCH}_{2}, \mathrm{~J}=2.2 \mathrm{~Hz}, \mathrm{~J}=7.1 \mathrm{~Hz}\right), 3.65\left(\mathrm{~s}, 2 \mathrm{H}, \mathrm{CH}_{2}\right)$, $1.35\left(\mathrm{t}, 3 \mathrm{H}, \mathrm{CH}_{3}, \mathrm{~J}=7.1 \mathrm{~Hz}\right), 1.23\left(\mathrm{~d}, 3 \mathrm{H}, \mathrm{CH}_{3}, \mathrm{~J}=6.7 \mathrm{~Hz}\right), 1.02\left(\mathrm{~d}, 3 \mathrm{H}, \mathrm{CH}_{3}, \mathrm{~J}=6.9 \mathrm{~Hz}\right) \mathrm{ppm} ;{ }^{13} \mathrm{C}$ NMR ( $\left.\mathrm{CDCl}_{3}, 100 \mathrm{MHz}\right) \delta 213.7$ (CS), 165.7 (CO), 152.2 (Car.), 149.7 (CHar.), 140.5 (CHar.), 133.5 (Car.), 123.3 (CHar.), $70.7\left(\mathrm{OCH}_{2}\right), 49.5(\mathrm{NCH}), 40.0\left(\mathrm{CH}_{2}\right), 21.9\left(\mathrm{CH}_{3}\right), 19.4$ $\left(\mathrm{CH}_{3}\right), 13.8\left(\mathrm{CH}_{3}\right)$ ppm. IR (neat) 2980, 2936, 1668, 1559, 1456, 1405, 1340, 1236, 1112 , $1051,752,731 \mathrm{~cm}^{-1}$. MS $\left(\mathrm{CI}+\mathrm{NH}_{3}\right) \mathrm{m} / \mathrm{z} 333,335\left(\mathrm{MH}^{+}\right)$.<smiles>CCOC(=S)SCC(=O)N(c1ccccc1Cl)C(C)(C)C</smiles>

Dithiocarbonic acid \{[tert-butyl-(2-chloro-phenyl)-carbamoyl]-methyl $\}$ ester ethyl ester 12 was prepared according to Method 3: The first reaction was carried out using a solution of the corresponding aniline $(410 \mathrm{mg}, 2.23 \mathrm{mmol})$ in dichloromethane $(20 \mathrm{~mL}), N, N$-dimethylaniline (0.26 mL, $2.05 \mathrm{mmol}, 0.9 \mathrm{eq})$ and chloroacetyl chloride (0.22 mL, $2.76 \mathrm{mmol}, 1.2 \mathrm{eq})$. The solution was refluxed until starting material was totally consumed. After work up, the crude residue was treated with Potassium $O$-Ethyl xanthate $(1.100 \mathrm{~g}, 6.87 \mathrm{mmol}, 3 \mathrm{eq})$ in acetone $(20 \mathrm{~mL})$ to give, after work up and purification by flash column chromatography (silica gel, petroleum ether-diethyl ether, 10:0 to 85:15), compound $\mathbf{1 2}$ in $\mathbf{8 1 \%}$ yield over 2 steps as a yellow oil. ${ }^{1} \mathrm{H}$ NMR $\left(\mathrm{CDCl}_{3}, 400 \mathrm{MHz}\right) \delta 7.53$ (m, 1H, CHar.), 7.35 (m, 3H, CHar.), 4.57 (q, $\left.2 \mathrm{H}, \mathrm{OCH}_{2}, \mathrm{~J}=7.1 \mathrm{~Hz}\right), 3.62\left(\mathrm{~d}, 1 \mathrm{H}, \mathrm{CH}_{2}, \mathrm{~J}=16.2 \mathrm{~Hz}\right), 3.54\left(\mathrm{~d}, 1 \mathrm{H}, \mathrm{CH}_{2}, \mathrm{~J}=16.2 \mathrm{~Hz}\right), 1.42(\mathrm{~s}, 9 \mathrm{H}$, $\left.\mathrm{CH}_{3}\right), 1.37\left(\mathrm{t}, 3 \mathrm{H}, \mathrm{CH}_{3}, \mathrm{~J}=7.1 \mathrm{~Hz}\right) \mathrm{ppm} ;{ }^{13} \mathrm{C} \mathrm{NMR}\left(\mathrm{CDCl}_{3}, 100 \mathrm{MHz}\right) \delta 214.1(\mathrm{CS}), 165.7$ (CO), 139.3 (Car.), 134.8 (Car.), 131.9 (CHar.), 130.6 (CHar.), 130.0 (CHar.), 127.9 (CHar.), $70.2\left(\mathrm{OCH}_{2}\right), 60.3(\mathrm{C}), 42.1\left(\mathrm{CH}_{2}\right), 28.7\left(\mathrm{CH}_{3}\right), 13.8\left(\mathrm{CH}_{3}\right) \mathrm{ppm}$. IR (neat) $2979,2935,1681$, $1584,1475,1438,1360,1222,1192,1147,1112,1057,768,741 \mathrm{~cm}^{-1} . \mathrm{MS}\left(\mathrm{CI}+\mathrm{NH}_{3}\right) \mathrm{m} / \mathrm{z}$ 346, $348\left(\mathrm{MH}^{+}\right)$. 
<smiles>CCOC(=S)SCC(=O)N(c1ccccc1C)C(C)(C)C</smiles>

Dithiocarbonic acid [(tert-butyl-o-tolyl-carbamoyl)-methyl] ester ethyl ester $\mathbf{1 4}$ was prepared according to Method 3: The first reaction was carried out using a solution of the corresponding aniline $(330 \mathrm{mg}, 2.02 \mathrm{mmol})$ in dichloromethane $(20 \mathrm{~mL}), N, N$-dimethylaniline (0.46 mL, $3.63 \mathrm{mmol}, 1.8 \mathrm{eq})$ and chloroacetyl chloride $(0.23 \mathrm{~mL}, 2.88 \mathrm{mmol}, 1.4 \mathrm{eq})$. The solution was stirred at r.t. until starting material was totally consumed. After work up, the crude residue was treated with Potassium $O$-Ethyl xanthate $(1.674 \mathrm{~g}, 10.5 \mathrm{mmol}, 5 \mathrm{eq})$ in acetone $(28 \mathrm{~mL})$ to yield, after work up and purification by flash column chromatography (silica gel, petroleum ether-diethyl ether, 10:0 to 85:15), 94\% of compound 14 over 2 steps as a yellow oil. ${ }^{1} \mathrm{H} \mathrm{NMR}\left(\mathrm{CDCl}_{3}, 400 \mathrm{MHz}\right) \delta 7.25(\mathrm{~m}, 3 \mathrm{H}, \mathrm{CHar}$.), 7.13 (dd, 1H, CHar., $\mathrm{J}=0.9 \mathrm{~Hz}, \mathrm{~J}=7.4 \mathrm{~Hz}), 4.54\left(\mathrm{q}, 2 \mathrm{H}, \mathrm{OCH}_{2}, \mathrm{~J}=7.1 \mathrm{~Hz}\right), 3.55\left(\mathrm{~d}, 1 \mathrm{H}, \mathrm{CH}_{2}, \mathrm{~J}=16.3 \mathrm{~Hz}\right), 3.43(\mathrm{~d}, 1 \mathrm{H}$, $\left.\mathrm{CH}_{2}, \mathrm{~J}=16.3 \mathrm{~Hz}\right), 2.34$ (s, 3H, CH$\left.H_{3}\right), 1.37\left(\mathrm{~s}, 9 \mathrm{H}, \mathrm{CH}_{3}\right), 1.33\left(\mathrm{t}, 3 \mathrm{H}, \mathrm{CH}_{3}, \mathrm{~J}=7.1 \mathrm{~Hz}\right) \mathrm{ppm} ;{ }^{13} \mathrm{C}$ NMR (CDCl, $100 \mathrm{MHz}) \delta 214.2$ (CS), 165.8 (CO), 140.5 (Car.), 137.1 (Car.), 131.5 (CHar.), 130.0 (CHar.), 128.8 (CHar.), 127.1 (CHar.), $70.2\left(\mathrm{OCH}_{2}\right), 59.5(C), 42.5\left(\mathrm{CH}_{2}\right), 28.8\left(\mathrm{CH}_{3}\right)$, $18.9\left(\mathrm{CH}_{3}\right), 13.8\left(\mathrm{CH}_{3}\right)$ ppm. IR (neat) 2977, 2934, 1659, 1491, 1456, 1361, 1230, 1193, $1113,1052,732 \mathrm{~cm}^{-1}$. MS $\left(\mathrm{CI}+\mathrm{NH}_{3}\right) \mathrm{m} / \mathrm{z} 326,328\left(\mathrm{MH}^{+}\right)$.<smiles>CCOC(=S)SCC(=O)N(c1ccccc1C(F)(F)F)C(C)(C)C</smiles>

Dithiocarbonic acid \{[tert-butyl-(2-trifluoromethyl-phenyl)-carbamoyl]-methyl $\}$ ester ethyl ester 19 was prepared according to Method 3: The first reaction was carried out using a solution of the corresponding aniline $(500 \mathrm{mg}, 2.30 \mathrm{mmol})$ in 1,2-dichloroethane $(15 \mathrm{~mL})$, $\mathrm{N}, \mathrm{N}$-dimethylaniline $(0.92 \mathrm{~mL}, 7.21 \mathrm{mmol}, 3.1 \mathrm{eq})$ and chloroacetyl chloride $(0.64 \mathrm{~mL}, 8.03$ mmol, $3.5 \mathrm{eq})$. The solution was refluxed until starting material was totally consumed. After work up, the crude residue was treated with Potassium $O$-Ethyl xanthate $(961 \mathrm{mg}, 6.00 \mathrm{mmol}$, $2.6 \mathrm{eq})$ in acetone $(25 \mathrm{~mL})$ to yield, after work up and purification by flash column chromatography (silica gel, petroleum ether-diethyl ether, 10:0 to 9:1), 53\% over 2 steps of compound 19 as a yellow oil. ${ }^{1} \mathrm{H}$ NMR $\left(\mathrm{CDCl}_{3}, 400 \mathrm{MHz}\right) \delta 7.79(\mathrm{dd}, 1 \mathrm{H}, \mathrm{CHar}$., J=1.1z, $\mathrm{J}=7.9 \mathrm{~Hz}$ ), 7.67 (dt, 1H, CHar., J=1.1Hz, J=7.6Hz), 7.56 (t, 1H, CHar., J=7.7Hz), 7.47 (d, 1H, CHar., J=7.8Hz), $4.55\left(\mathrm{~m}, 2 \mathrm{H}, \mathrm{OCH}_{2}, \mathrm{~J}=7.1 \mathrm{~Hz}\right), 3.60\left(\mathrm{~d}, 1 \mathrm{H}, \mathrm{CH}_{2}, \mathrm{~J}=16.0 \mathrm{~Hz}\right), 3.51(\mathrm{~d}, 1 \mathrm{H}$, $\left.\mathrm{CH}_{2}, \mathrm{~J}=16.0 \mathrm{~Hz}\right), 1.40\left(\mathrm{~s}, 9 \mathrm{H}, \mathrm{CH}_{3}\right), 1.35\left(\mathrm{t}, 1 \mathrm{H}, \mathrm{CH}_{3}, \mathrm{~J}=7.1 \mathrm{~Hz}\right) \mathrm{ppm} ;{ }^{13} \mathrm{C} \mathrm{NMR}\left(\mathrm{CDCl}_{3}, 100\right.$ MHz) $\delta 214.1$ (s, CS), 166.3 (s, CO), 139.4 (q, Car., J=1.8Hz), 133.1 (s, CHar.), 132.5 (s,

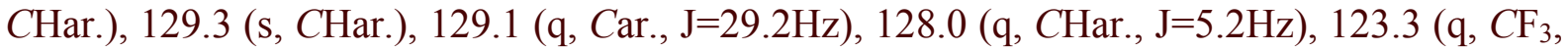
$\mathrm{J}=273.8 \mathrm{~Hz}), 70.4\left(\mathrm{~s}, \mathrm{OCH}_{2}\right), 60.8(\mathrm{~s}, C), 42.4\left(\mathrm{q}, \mathrm{CH}_{2}, \mathrm{~J}=1.4 \mathrm{~Hz}\right), 28.5\left(\mathrm{~s}, \mathrm{CH}_{3}\right), 13.8\left(\mathrm{~s}, \mathrm{CH}_{3}\right)$ ppm. IR (neat) 2981, 2936, 1668, 1494, 1452, 1396, 1344, 1313, 1232, 1173, 1125, 1111, $1052,1036,769 \mathrm{~cm}^{-1}$. MS $\left(\mathrm{CI}+\mathrm{NH}_{3}\right) \mathrm{m} / \mathrm{z} 379\left(\mathrm{MH}^{+}\right)$. 
<smiles>CC(C)(C)N(C(=O)CCl)c1c(Cl)cccc1Cl</smiles>

$N$-tert-Butyl-2-chloro- $N$-(2,6-dichloro-phenyl)-acetamide 21a was prepared according to the following procedure: To a stirred solution of tert-Butyl-(2,6-dichloro-phenyl)-amine (630 $\mathrm{mg}, 2.89 \mathrm{mmol})$ in 1,2-dichloroethane $(15 \mathrm{~mL})$ at r.t. under argon, were successively added $N, N$-dimethylaniline $(0.74 \mathrm{~mL}, 5.80 \mathrm{mmol}, 2$ eq) and chloroacetyl chloride $(0.35 \mathrm{~mL}, 4.39$ mmol, $1.5 \mathrm{eq})$. The solution was stirred under reflux overnight until starting material was totally consumed. The crude mixture was poured in saturated aqueous $\mathrm{Na}_{2} \mathrm{CO}_{3}$, extracted with dichloromethane, dried and concentrated under reduced pressure. The resulting crude residue was purified by flash column chromatography (silica gel, petroleum ether-diethyl ether, 10:0 to $93: 7)$ to give compound $\mathbf{2 1 a}$ in $\mathbf{9 4 \%}$ yield as white crystals. $\left(\right.$ m.p. $=91-94^{\circ} \mathrm{C}$, recrystallized with heptane/ethyl acetate). ${ }^{1} \mathrm{H}$ NMR $\left(\mathrm{CDCl}_{3}, 400 \mathrm{MHz}\right) \delta 7.43$ (dd, $2 \mathrm{H}, \mathrm{CHar} ., \mathrm{J}=7.6 \mathrm{~Hz}$, $\mathrm{J}=8.6 \mathrm{~Hz}), 7.28(\mathrm{dd}, 1 \mathrm{H}, \mathrm{CHar} ., \mathrm{J}=7.6 \mathrm{~Hz}, \mathrm{~J}=8.6 \mathrm{~Hz}), 3.60\left(\mathrm{~s}, 2 \mathrm{H}, \mathrm{CH}_{2}\right), 1.44\left(\mathrm{~s}, 9 \mathrm{H}, \mathrm{CH}_{3}\right) \mathrm{ppm}$ ; ${ }^{13} \mathrm{C} \mathrm{NMR}\left(\mathrm{CDCl}_{3}, 100 \mathrm{MHz}\right) \delta 164.7$ (CO), 136.8 (Car.), 136.7 (Car.), 130.3 (CHar.), 129.3 (CHar.), $61.9(C), 44.5\left(\mathrm{CH}_{2}\right), 28.4\left(\mathrm{CH}_{3}\right)$ ppm. IR $\left(\mathrm{CH}_{2} \mathrm{Cl}_{2}\right)$ 2976, 1686, 1604, 1561, 1445, $1435,1422,1352,1290,1236,1188,1080 \mathrm{~cm}^{-1} . \mathrm{MS}\left(\mathrm{CI}+\mathrm{NH}_{3}\right) \mathrm{m} / \mathrm{z} 295,297,299\left(\mathrm{MH}^{+}\right)$.<smiles>CC(C)(C)COC(=S)SCC(=O)N(c1c(Cl)cccc1Cl)C(C)(C)C</smiles>

Dithiocarbonic acid $\{[$ tert-butyl-(2,6-dichloro-phenyl)-carbamoyl $]$-methyl $\}$ ester (2,2dimethyl-propyl) ester 22a was prepared according to the following procedure : To a solution of $N$-tert-Butyl-2-chloro- $N$-(2,6-dichloro-phenyl)-acetamide $(400 \mathrm{mg}, 1.36 \mathrm{mmol})$ in acetonitrile $(2 \mathrm{~mL})$ under argon was added Sodium $O$-Neopentyl xanthate ${ }^{2}(603 \mathrm{mg}, 3.24$ mmol, $2.4 \mathrm{eq}$ ) and the reaction mixture was refluxed for 6 hours. After consumption of all the starting material, the resulting mixture was diluted with water, extracted with ether, washed several times with brine, dried and concentrated. The residue was purified by flash column chromatography (silica gel, petroleum ether-diethyl ether, 10:0 to 95:5) to give compound 22a in $\mathbf{8 7 \%}$ yield as white crystals. (m.p. $=135-138^{\circ} \mathrm{C}$, recrystallized with heptane/ethyl acetate). ${ }^{1} \mathrm{H}$ NMR $\left(\mathrm{CDCl}_{3}, 400 \mathrm{MHz}\right) \delta 7.47$ (dd, 2H, CHar., J=7.7Hz, J=8.4Hz), 7.29 (dd, 1H CHar., $\mathrm{J}=7.7 \mathrm{~Hz}, \mathrm{~J}=8.4 \mathrm{~Hz}), 4.15\left(\mathrm{~s}, 2 \mathrm{H}, \mathrm{OCH}_{2}\right), 3.67\left(\mathrm{~s}, 2 \mathrm{H}, \mathrm{CH}_{2}\right), 1.48\left(\mathrm{~s}, 9 \mathrm{H}, \mathrm{CH}_{3}\right), 0.92(\mathrm{~s}, 9 \mathrm{H}$, $\left.\mathrm{CH}_{3}\right) \mathrm{ppm} ;{ }^{13} \mathrm{C} \mathrm{NMR}\left(\mathrm{CDCl}_{3}, 100 \mathrm{MHz}\right) \delta 213.7$ (CS), 165.6 (CO), 137.9 (Car.), 136.9 (Car.), 130.1 (CHar.), 129.3 (CHar.), $83.4\left(\mathrm{OCH}_{2}\right), 61.8(C), 42.0\left(\mathrm{CH}_{2}\right), 31.8(\mathrm{C}), 28.6$ $\left(\mathrm{CH}_{3}\right), 26.6\left(\mathrm{CH}_{3}\right)$ ppm. IR $\left(\mathrm{CH}_{2} \mathrm{Cl}_{2}\right) 2965,2938,1666,1561,1445,1436,1425,1366,1353$, 1284, 1248, 1232, 1188, 1070, $1030 \mathrm{~cm}^{-1}$. MS $\left(\mathrm{CI}+\mathrm{NH}_{3}\right) \mathrm{m} / \mathrm{z} 423,425\left(\mathrm{MH}^{+}\right)$. Anal. calcd. for $\mathrm{C}_{18} \mathrm{H}_{25} \mathrm{Cl}_{2} \mathrm{NO}_{2} \mathrm{~S}_{2}$ : C, 51.18; H, 5.97, found: C, 50.71; H, 5.94.<smiles>CN1C(=O)Cc2ccnc(Cl)c21</smiles> 
7-Chloro-1-methyl-1,3-dihydro-pyrrolo[2,3-c]pyridin-2-one 4c was prepared according to Method 4 using a solution of the corresponding xanthate $(421 \mathrm{mg}, 1.38 \mathrm{mmol})$ in chlorobenzene $(0.1 \mathrm{~mol} / \mathrm{L})$. Lauroyl peroxide $(685 \mathrm{mg}, 1.72 \mathrm{mmol}, 1.2 \mathrm{eq})$ was then added portionwise (20 mol \% per 20 minutes) to the refluxing solution. The crude mixture was then cooled to room temperature, concentrated under reduced pressure and purified by flash column chromatography (silica gel, petroleum ether-diethyl ether, 10:0 to 3:7) to give compound $4 \mathbf{c}$ in $\mathbf{3 9 \%}$ yield as white crystals. (m.p. $=152-155^{\circ} \mathrm{C}$, recrystallized with heptane/ethyl acetate). ${ }^{1} \mathrm{H}$ NMR $\left(\mathrm{CDCl}_{3}, 400 \mathrm{MHz}\right) \delta 8.10(\mathrm{~d}, 1 \mathrm{H}, \mathrm{CHar} ., \mathrm{J}=4.6 \mathrm{~Hz}), 7.15(\mathrm{~d}$, $1 \mathrm{H}, \mathrm{CHar} ., \mathrm{J}=4.6 \mathrm{~Hz}$ ), 3.56 (bs, $\left.2 \mathrm{H}, \mathrm{CH}_{2}\right), 3.55$ (s, 3H, $\left.\mathrm{CH}_{3}\right) \mathrm{ppm} ;{ }^{13} \mathrm{C} \mathrm{NMR}\left(\mathrm{CDCl}_{3}, 100\right.$ MHz) $\delta 173.6$ (CO), 143.5 (CHar.), 139.1 (Car.), 136.7 (Car.), 131.6 (Car.), 118.8 (CHar.), $35.6\left(\mathrm{CH}_{2}\right), 29.3\left(\mathrm{CH}_{3}\right) \mathrm{ppm}$. IR $\left(\mathrm{CH}_{2} \mathrm{Cl}_{2}\right)$ 3063, 2986, 2954, 2927, 1728, 1676, 1563, 1469, $1428,1411,1359,1299,1232,1186,1113,1049 \mathrm{~cm}^{-1} . \mathrm{MS}\left(\mathrm{CI}+\mathrm{NH}_{3}\right) \mathrm{m} / \mathrm{z} 183,185\left(\mathrm{MH}^{+}\right)$. Anal. calcd. for $\mathrm{C}_{8} \mathrm{H}_{7} \mathrm{ClN}_{2} \mathrm{O}_{2}$ : C, 52.62; H, 3.86, found: C, 52.46; H, 3.84.<smiles>CC(C)(C)NC(=O)Cc1ccccc1Cl</smiles>

$N$-tert-Butyl-2-(2-chloro-phenyl)-acetamide 13 was prepared according to Method 4 using a solution of the corresponding xanthate $(320 \mathrm{mg}, 0.92 \mathrm{mmol})$ in octane $(20 \mathrm{~mL})$. Lauroyl peroxide (330 mg, $0.83 \mathrm{mmol}, 0.9 \mathrm{eq}$ ) was then added portionwise (20 mol \% per 20 minutes) to the refluxing solution. The crude mixture was then cooled to room temperature, concentrated under reduced pressure and purified by flash column chromatography (silica gel, petroleum ether-diethyl ether, 10:0 to 7:3) to give compound $\mathbf{1 3}$ in $\mathbf{7 3 \%}$ yield as white crystals. (m.p. $=138-141^{\circ} \mathrm{C}$, recrystallized with heptane/ethyl acetate). ${ }^{1} \mathrm{H}$ NMR $\left(\mathrm{CDCl}_{3}, 400\right.$ MHz) $\delta$ 7.32-7.15 (m, 4H, CHar.), 5.25 (bs, 1H, NH), 3.51 (s, 2H, $\left.\mathrm{CH}_{2}\right), 1.23$ (s, 9H, $\left.\mathrm{CH}_{3}\right)$ ppm; ${ }^{13} \mathrm{C} \mathrm{NMR}\left(\mathrm{CDCl}_{3}, 100 \mathrm{MHz}\right) \delta 168.9$ (CO), 134.4 (Car.), 133.7 (Car.), 131.7 (CHar.), 129.8 (CHar.), 128.8 (CHar.), 127.3 (CHar.), $51.4(C), 42.7\left(\mathrm{CH}_{2}\right), 28.7\left(\mathrm{CH}_{3}\right)$ ppm. IR $\left(\mathrm{CH}_{2} \mathrm{Cl}_{2}\right) 3426,3043,2969,1674,1513,1474,1454,1365,1221,1123,1054 \mathrm{~cm}^{-1} . \mathrm{MS}$ $\left(\mathrm{CI}+\mathrm{NH}_{3}\right) \mathrm{m} / \mathrm{z} 226,228\left(\mathrm{MH}^{+}\right)$. Anal. calcd. for $\mathrm{C}_{12} \mathrm{H}_{16} \mathrm{ClNO}: \mathrm{C}, 63.86 ; \mathrm{H}, 7.14$, found: $\mathrm{C}$, $63.68 ; \mathrm{H}, 7.11$.<smiles>CC(C)N1C(=O)Cc2ccnc(Cl)c21</smiles>

7-Chloro-1-isopropyl-1,3-dihydro-pyrrolo[2,3-c]pyridin-2-one 4d was prepared according to Method 4 using a solution of the corresponding xanthate $(998 \mathrm{mg}, 3.01 \mathrm{mmol})$ in chlorobenzene $(30 \mathrm{~mL})$. Lauroyl peroxide $(1.260 \mathrm{~g}, 3.16 \mathrm{mmol}, 1.0 \mathrm{eq})$ was then added portionwise (20 mol \% per 20 minutes) to the refluxing solution. The crude mixture was then cooled to room temperature, concentrated under reduced pressure and purified by flash column chromatography (silica gel, petroleum ether-diethyl ether, 10:0 to 4:6) to give compound $\mathbf{4 d}$ in $\mathbf{1 8 \%}$ yield as orange crystals. (m.p. $=148-151{ }^{\circ} \mathrm{C}$, recrystallized with heptane/ethyl acetate). ${ }^{1} \mathrm{H}$ NMR $\left(\mathrm{CDCl}_{3}, 400 \mathrm{MHz}\right) \delta 8.07$ (d, 1H, CHar., J=4.5Hz), 7.12 (d, $1 \mathrm{H}, \mathrm{CHar}$., J=4.5Hz), 5.20 (sept., $1 \mathrm{H}, \mathrm{CH}, \mathrm{J}=6.9 \mathrm{~Hz}$ ), 3.48 (s, $\left.2 \mathrm{H}, \mathrm{CH}_{2}\right), 1.55$ (d, $6 \mathrm{H}, \mathrm{CH}_{3}$, $\mathrm{J}=6.9 \mathrm{~Hz}) \mathrm{ppm} ;{ }^{13} \mathrm{C} \mathrm{NMR}\left(\mathrm{CDCl}_{3}, 100 \mathrm{MHz}\right) \delta 174.2$ (CO), 143.0 (CHar.), 139.1 (Car.), 137.6 (Car.), 130.8 (Car.), 118.8 (CHar.), 47.4 (b, $\mathrm{CH}), 36.2\left(\mathrm{CH}_{2}\right), 19.9\left(\mathrm{CH}_{3}\right)$ ppm. IR $\left(\mathrm{CH}_{2} \mathrm{Cl}_{2}\right)$ 2977, 1720, 1559, 1447, 1424, 1379, 1343, 1270, 1215, 1180, 1164, 1120, $1077 \mathrm{~cm}^{-1}$. MS $\left(\mathrm{CI}+\mathrm{NH}_{3}\right) \mathrm{m} / \mathrm{z} 211,213\left(\mathrm{MH}^{+}\right)$. Anal. calcd. for $\mathrm{C}_{10} \mathrm{H}_{11} \mathrm{ClN}_{2} \mathrm{O}: \mathrm{C}, 57.02 ; \mathrm{H}, 5.26$, found: $\mathrm{C}$, $57.17 ; \mathrm{H}, 5.23$. 
<smiles>CC(C)NC(=O)Cc1cccnc1Cl</smiles>

2-(2-Chloro-pyridin-3-yl)- $N$-isopropyl-acetamide 5d was obtained as well in $\mathbf{3 1 \%}$ yield as white crystals. (m.p. $=139-142^{\circ} \mathrm{C}$, recrystallized with heptane/ethyl acetate). ${ }^{1} \mathrm{H}$ NMR $\left(\mathrm{CDCl}_{3}, 400 \mathrm{MHz}\right) \delta 8.30$ (dd, 1H, CHar., J=1.9Hz, J=4.8Hz), 7.72 (dd, 1H, CHar., J=1.9Hz, $\mathrm{J}=7.5 \mathrm{~Hz}), 7.24(\mathrm{dd}, 1 \mathrm{H}, \mathrm{CHar} ., \mathrm{J}=4.8 \mathrm{~Hz}, \mathrm{~J}=7.5 \mathrm{~Hz}), 5.61(\mathrm{~s}, 1 \mathrm{H}, \mathrm{NH}), 4.07(\mathrm{~m}, 1 \mathrm{H}, \mathrm{NCH})$, $3.59\left(\mathrm{~s}, 2 \mathrm{H}, \mathrm{CH}_{2}\right), 1.13\left(\mathrm{~d}, 6 \mathrm{H}, \mathrm{CH}_{3}, \mathrm{~J}=6.6 \mathrm{~Hz}\right) \mathrm{ppm} ;{ }^{13} \mathrm{C} \mathrm{NMR}\left(\mathrm{CDCl}_{3}, 100 \mathrm{MHz}\right) \delta 167.7$ (CO), 151.3 (Car.), 148.5 (CHar.), 140.3 (CHar.), 130.2 (Car.), 123.0 (CHar.), 41.9 (CH), $41.0\left(\mathrm{CH}_{2}\right), 22.7\left(\mathrm{CH}_{3}\right)$ ppm. IR $\left(\mathrm{CH}_{2} \mathrm{Cl}_{2}\right)$ 3683, 3429, 2981, 2932, 1672, 1583, 1565, 1514, 1457, 1411, 1388, 1172, 1128, $1075 \mathrm{~cm}^{-1}$. MS $\left(\mathrm{CI}+\mathrm{NH}_{3}\right) \mathrm{m} / \mathrm{z} 213,215\left(\mathrm{MH}^{+}\right)$. Anal. calcd. for $\mathrm{C}_{10} \mathrm{H}_{13} \mathrm{ClN}_{2} \mathrm{O}$ : C, 56.47; H, 6.16, found: C, 56.26; H, 5.82.<smiles>CC(C)(C)NC(=O)Cc1ccccc1C(F)(F)F</smiles>

$N$-tert-Butyl-2-(2-trifluoromethyl-phenyl)-acetamide 20 was prepared according to Method 4 using a solution of the corresponding xanthate $(280 \mathrm{mg}, 0.74 \mathrm{mmol})$ in octane $(0.09 \mathrm{~mol} / \mathrm{L})$. Lauroyl peroxide (184 mg, $0.46 \mathrm{mmol}, 0.6 \mathrm{eq}$ ) was then added portionwise ( $20 \mathrm{~mol} \%$ per 20 minutes usually) to the refluxing solution. The crude mixture was then cooled to room temperature, concentrated under reduced pressure and purified by flash column chromatography (silica gel, petroleum ether-diethyl ether, 10:0 to 8:2) to give compound 20 in $\mathbf{4 6 \%}$ yield as white crystals. (m.p. $=108-111^{\circ} \mathrm{C}$, recrystallized with heptane/ethyl acetate). ${ }^{1} \mathrm{H}$ NMR $\left(\mathrm{CDCl}_{3}, 400 \mathrm{MHz}\right) \delta 7.67$ (d, 1H, CHar., J=7.7Hz), 7.54 (t, 1H, CHar., J=7.5Hz), 7.47 (d, 1H, CHar., J=7.5Hz), 7.39 (t, 1H, CHar., J=7.7Hz), 5.22 (bs, 1H, NH), 3.64 (s, 2H, $\mathrm{CH}_{2}$ ), 1.30 (s, 9H, $\left.\mathrm{CH}_{3}\right)$ ppm; ${ }^{13} \mathrm{C} \mathrm{NMR}\left(\mathrm{CDCl}_{3}, 100 \mathrm{MHz}\right) \delta 167.0$ (s, CO), 133.8 (q, Car., $\mathrm{J}=1.6 \mathrm{~Hz}$ ), 132.6 (s, CHar.), 132.2 (q, CHar., J=0.8Hz), 128.7 (q, Car., J=30.0Hz), 127.4 (s, CHar.), 126.2 (q, CHar., J=5.6Hz), 124.5 (q, $\left.C F_{3}, \mathrm{~J}=273.7 \mathrm{~Hz}\right), 51.5$ (s, $\mathrm{CH}_{2}$ ), 41.5 (q, $\mathrm{CH}_{2}$, $\mathrm{J}=1.7 \mathrm{~Hz}), 28.7\left(\mathrm{~s}, \mathrm{CH}_{3}\right) \mathrm{ppm}$. IR $\left(\mathrm{CH}_{2} \mathrm{Cl}_{2}\right) 3430,3058,2969,2931,1678,1514,1453,1393$, $1315,1220,1182,1160,1122,1060,1038 \mathrm{~cm}^{-1}$. MS $\left(\mathrm{CI}+\mathrm{NH}_{3}\right) \mathrm{m} / \mathrm{z} 260\left(\mathrm{MH}^{+}\right)$. Anal. calcd. for $\mathrm{C}_{13} \mathrm{H}_{16} \mathrm{~F}_{3} \mathrm{NO}$ : $\mathrm{C}, 60.22 ; \mathrm{H}, 6.22$, found: $\mathrm{C}, 59.91 ; \mathrm{H}, 6.29$.<smiles>CC(C)(C)NC(=O)Cc1c(Cl)cccc1Cl</smiles>

$N$-tert-Butyl-2-(2,6-dichloro-phenyl)-acetamide 23a was prepared according to Method 4 using a solution of the corresponding xanthate $(50 \mathrm{mg}, 0.12 \mathrm{mmol})$ in di- $n$-butylether $(0.08$ $\mathrm{mol} / \mathrm{L}$ ). Lauroyl peroxide (30 mg, $0.07 \mathrm{mmol}, 0.6 \mathrm{eq}$ ) was then added portionwise $(20 \mathrm{~mol} \%$ per 20 minutes usually) to the refluxing solution. The crude mixture was then cooled to room temperature, concentrated under reduced pressure and purified by flash column chromatography (silica gel, petroleum ether) to give in $\mathbf{9 8 \%}$ yield compound $\mathbf{2 3 a}$ as white crystals. (m.p. $=168-171^{\circ} \mathrm{C}$, recrystallized with heptane/ethyl acetate). ${ }^{1} \mathrm{H}$ NMR $\left(\mathrm{CDCl}_{3}, 400\right.$ $\mathrm{MHz}) \delta 7.33$ (d, 2H, CHar., J=8.1Hz), 7.16 (dd, 1H, CHar., J=7.7Hz, J=8.4Hz), 5.28 (s, 1H, $\mathrm{NH}), 3.84\left(\mathrm{~s}, 2 \mathrm{H}, \mathrm{CH}_{2}\right), 1.31$ (s, 9H, $\left.\mathrm{CH}_{3}\right) \mathrm{ppm} ;{ }^{13} \mathrm{C} \mathrm{NMR}\left(\mathrm{CDCl}_{3}, 100 \mathrm{MHz}\right) \delta 167.5(\mathrm{CO})$, 136.3 (Car.), 132.2 (Car.), 129.0 (CHar.), 128.4 (CHar.), $51.6(C), 39.9\left(\mathrm{CH}_{2}\right), 28.7\left(\mathrm{CH}_{3}\right)$ ppm. IR $\left(\mathrm{CH}_{2} \mathrm{Cl}_{2}\right)$ 3682, 3424, 3048, 2954, 1678, 1563, 1513, 1453, 1393, 1367, 1219, 1088 $\mathrm{cm}^{-1}$. MS $\left(\mathrm{CI}+\mathrm{NH}_{3}\right) \mathrm{m} / \mathrm{z} 260,262,264\left(\mathrm{MH}^{+}\right)$. Anal. calcd. for $\mathrm{C}_{12} \mathrm{H}_{15} \mathrm{Cl}_{2} \mathrm{NO}: \mathrm{C}, 55.40 ; \mathrm{H}$, 5.81, found: C, 55.18; H, 5.83 . 
<smiles>Clc1cccc(Cl)c1CCc1c(Cl)cccc1Cl</smiles>

2,6,2',6'-Tetrachloro-bibenzyl 24 was prepared according to Method 4 using a solution of the corresponding xanthate $(100 \mathrm{mg}, 0.24 \mathrm{mmol})$ in chlorobenzene $(0.47 \mathrm{~mol} / \mathrm{L})$. Lauroyl peroxide (119 mg, $0.30 \mathrm{mmol}, 1.2 \mathrm{eq}$ ) was then added portionwise (20 mol \% per 20 minutes usually) to the refluxing solution. The crude mixture was then cooled to room temperature, concentrated under reduced pressure and purified by flash column chromatography (silica gel, petroleum ether) to give in $\mathbf{2 6 \%}$ yield a pure fraction of compound $\mathbf{2 4}$ as white crystals. (m.p. $=155-157^{\circ} \mathrm{C}$, recrystallized with heptane/ethyl acetate). Its spectroscopic properties were identical to those described in the literature. ${ }^{6}{ }^{1} \mathrm{H}$ NMR $\left(\mathrm{CDCl}_{3}, 400 \mathrm{MHz}\right) \delta 7.28(\mathrm{~d}, 4 \mathrm{H}$, CHar., J=7.9Hz), 7.09 (dd, 2H, CHar., J=7.6Hz, J=8.4Hz), 3.26 (s, 4H, CH ) ppm; ${ }^{13} \mathrm{C}$ NMR $\left(\mathrm{CDCl}_{3}, 100 \mathrm{MHz}\right) \delta 136.8$ (Car.), 136.0 (Car.), 128.3 (CHar.), 128.0 (CHar.), $29.8\left(\mathrm{CH}_{2}\right)$ ppm. IR $\left(\mathrm{CH}_{2} \mathrm{Cl}_{2}\right) 3056,2956,2928,1582,1562,1453,1436,1272,1118,1086,1070 \mathrm{~cm}^{-1}$. $\mathrm{MS}\left(\mathrm{CI}+\mathrm{NH}_{3}\right) \mathrm{m} / \mathrm{z} 318,320,322,324\left(\mathrm{MH}^{+}\right)$.<smiles>CC(C)(C)NC(=O)Cc1cccnc1Cl</smiles>

$N$-tert-Butyl-2-(2-chloro-pyridin-3-yl)-acetamide 5a was prepared according to Method 4 using a solution of the corresponding xanthate $(368 \mathrm{mg}, 1.06 \mathrm{mmol})$ in octane $(0.08 \mathrm{~mol} / \mathrm{L})$. Lauroyl peroxide ( $258 \mathrm{mg}, 0.65 \mathrm{mmol}, 0.6 \mathrm{eq}$ ) was then added portionwise ( $20 \mathrm{~mol} \%$ per 20 minutes usually) to the refluxing solution. The crude mixture was then cooled to room temperature, concentrated under reduced pressure and purified by flash column chromatography (silica gel, petroleum ether-diethyl ether, 10:0 to 3:7) to give compound 5a in $\mathbf{7 1 \%}$ yield as white crystals. (m.p. $=140-142^{\circ} \mathrm{C}$, recrystallized with heptane/ethyl acetate). ${ }^{1} \mathrm{H} \mathrm{NMR}\left(\mathrm{CDCl}_{3}, 400 \mathrm{MHz}\right) \delta 8.28(\mathrm{dd}, 1 \mathrm{H}, \mathrm{CHar} ., \mathrm{J}=1.7 \mathrm{~Hz}, \mathrm{~J}=4.7 \mathrm{~Hz}), 7.69$ (dd, 1H, CHar., $\mathrm{J}=1.7 \mathrm{~Hz}, \mathrm{~J}=7.5 \mathrm{~Hz}$ ), 7.21 (dd, 1H, CHar., $\mathrm{J}=4.7 \mathrm{~Hz}, \mathrm{~J}=7.5 \mathrm{~Hz}), 5.63$ (bs, 1H, NH), 3.53 (s, 2H, $\left.\mathrm{CH}_{2}\right), 1.31$ (s, 9H, $\left.\mathrm{CH}_{3}\right)$ ppm; ${ }^{13} \mathrm{C} \mathrm{NMR}\left(\mathrm{CDCl}_{3}, 100 \mathrm{MHz}\right) \delta 167.9$ (CO), 151.3 (Car.), 148.3 (CHar.), 140.2 (CHar.), 130.5 (Car.), 122.9 (CHar.), $51.7(C), 41.7\left(\mathrm{CH}_{2}\right), 28.7\left(\mathrm{CH}_{3}\right) \mathrm{ppm}$. IR $\left(\mathrm{CH}_{2} \mathrm{Cl}_{2}\right)$ 3666, 3430, 3049, 2969, 2930, 1674, 1583, 1566, 1513, 1453, 1409, 1366, 1335, $1222,1075 \mathrm{~cm}^{-1}$. MS $\left(\mathrm{CI}+\mathrm{NH}_{3}\right) \mathrm{m} / \mathrm{z} 227,229\left(\mathrm{MH}^{+}\right)$. Anal. calcd. for $\mathrm{C}_{11} \mathrm{H}_{15} \mathrm{ClN}_{2} \mathrm{O}: \mathrm{C}, 58.28$; $\mathrm{H}, 6.67$, found: $\mathrm{C}, 58.38 ; \mathrm{H}, 6.71$.<smiles>CC(C)(C)NC(=O)Cc1ccc(Br)nc1Cl</smiles>

2-(6-Bromo-2-chloro-pyridin-3-yl)- $N$-tert-butyl-acetamide $\mathbf{5 b}$ was prepared according to Method 4 using a solution of the corresponding xanthate $(244 \mathrm{mg}, 0.57 \mathrm{mmol})$ in octane $(0.1$ $\mathrm{mol} / \mathrm{L}$ ). Lauroyl peroxide (136 mg, $0.34 \mathrm{mmol}, 0.6 \mathrm{eq}$ ) was then added portionwise $(20 \mathrm{~mol} \%$ per 20 minutes usually) to the refluxing solution. The crude mixture was then cooled to room temperature, concentrated under reduced pressure and purified by flash column chromatography (silica gel, petroleum ether-diethyl ether, 10:0 to 6:4) to give compound $\mathbf{5 b}$ in $\mathbf{7 1 \%}$ yield as white crystals. (m.p. $=158-162^{\circ} \mathrm{C}$, recrystallized with heptane/ethyl acetate). ${ }^{1} \mathrm{H}$ NMR $\left(\mathrm{CDCl}_{3}, 400 \mathrm{MHz}\right) \delta 7.59$ (d, 1H, CHar., J=7.9Hz), 7.42 (d, 1H, CHar., J=7.9Hz), $5.54(\mathrm{~s}, 1 \mathrm{H}, \mathrm{NH}), 3.50\left(\mathrm{~s}, 2 \mathrm{H}, \mathrm{CH}_{2}\right), 1.34\left(\mathrm{~s}, 9 \mathrm{H}, \mathrm{CH}_{3}\right) \mathrm{ppm} ;{ }^{13} \mathrm{C} \mathrm{NMR}\left(\mathrm{CDCl}_{3}, 100 \mathrm{MHz}\right) \delta$

\footnotetext{
${ }^{6}$ (1) McBay H., Tucker, O., Groves, P. T. J. Org. Chem. 1959, 24, 536-545. (2) Suh, Y., Lee, J., Kim, S., Rieke,
} R.D. J. Organomet. Chem. 2003, 684, 1-2, 20-36. (3) Huang, H. H. Aust. J. Chem. 1976, 29, 2415-2422. 
167.3 (CO), 150.3 (Car.), 142.2 (CHar.), 138.8 (Car.), 129.7 (Car.), 127.3 (CHar.), 52.0 (C), $41.0\left(\mathrm{CH}_{2}\right), 28.8\left(\mathrm{CH}_{3}\right)$ ppm. IR $\left(\mathrm{CH}_{2} \mathrm{Cl}_{2}\right) 3430,3048,2969,2932,1678,1575,1545,1513$, $1454,1428,1418,1393,1365,1357,1218,1122,1070 \mathrm{~cm}^{-1} . \mathrm{MS}\left(\mathrm{CI}+\mathrm{NH}_{3}\right) \mathrm{m} / \mathrm{z} 305,307,309$ $\left(\mathrm{MH}^{+}\right)$.<smiles>O=C(Cc1cccnc1Cl)NC1CCCCC1</smiles>

2-(2-Chloro-pyridin-3-yl)- $N$-cyclohexyl-acetamide 5e was prepared according to Method 4 using a solution of the corresponding xanthate $(1.185 \mathrm{~g}, 3.18 \mathrm{mmol})$ in chlorobenzene $(31$ $\mathrm{mL})$. Lauroyl peroxide $(1.523 \mathrm{~g}, 3.82 \mathrm{mmol}, 1.2 \mathrm{eq})$ was then added portionwise $(20 \mathrm{~mol} \%$ per 20 minutes) to the refluxing solution. The crude mixture was then cooled to room temperature, concentrated under reduced pressure and purified by flash column chromatography (silica gel, petroleum ether-diethyl ether, 10:0 to 0:10) to give compound 5e in $\mathbf{4 2 \%}$ yield as white crystals. (m.p. $=140-143^{\circ} \mathrm{C}$, recrystallized with heptane/ethyl acetate). ${ }^{1} \mathrm{H}$ NMR $\left(\mathrm{CDCl}_{3}, 400 \mathrm{MHz}\right) \delta 8.33(\mathrm{dd}, 1 \mathrm{H}, \mathrm{CHar} ., \mathrm{J}=1.9 \mathrm{~Hz}, \mathrm{~J}=4.8 \mathrm{~Hz}), 7.75$ (dd, $1 \mathrm{H}, \mathrm{CHar}$, $\mathrm{J}=1.9 \mathrm{~Hz}, \mathrm{~J}=7.5 \mathrm{~Hz}$ ), 7.26 (dd, 1H, CHar., $\mathrm{J}=4.8 \mathrm{~Hz}, \mathrm{~J}=7.5 \mathrm{~Hz}), 5.47$ (bs, $1 \mathrm{H}, \mathrm{NH}), 3.78(\mathrm{~m}, 1 \mathrm{H}$, $\mathrm{NCH}), 3.62$ (s, 2H, $\left.\mathrm{CH}_{2}\right), 1.92-1.89\left(\mathrm{~m}, 2 \mathrm{H}, \mathrm{CH}_{2}\right), 1.71-1.58\left(\mathrm{~m}, 3 \mathrm{H}, \mathrm{CH}_{2}\right), 1.40-1.25(\mathrm{~m}$, $\left.2 \mathrm{H}, \mathrm{CH}_{2}\right), 1.20-1.07\left(\mathrm{~m}, 3 \mathrm{H}, \mathrm{CH}_{2}\right) \mathrm{ppm} ;{ }^{13} \mathrm{C} \mathrm{NMR}\left(\mathrm{CDCl}_{3}, 100 \mathrm{MHz}\right) \delta 167.6(\mathrm{CO}), 151.4$ (Car.), 148.6 CHar.), 140.3 (CHar.), 130.3 (Car.), 123.0 (CHar.), $48.7(\mathrm{CH}), 41.2\left(\mathrm{CH}_{2}\right), 33.1$ $\left(\mathrm{CH}_{2}\right), 25.6\left(\mathrm{CH}_{2}\right), 24.8\left(\mathrm{CH}_{2}\right)$ ppm. IR $\left(\mathrm{CH}_{2} \mathrm{Cl}_{2}\right)$ 3427, 3054, 2935, 2855, 1671, 1565, 1514, 1450, 1410, 1338, 1279, 1156, 1127, $1075 \mathrm{~cm}^{-1}$. MS $\left(\mathrm{CI}+\mathrm{NH}_{3}\right) \mathrm{m} / \mathrm{z} 253,255\left(\mathrm{MH}^{+}\right)$. Anal. calcd. for $\mathrm{C}_{13} \mathrm{H}_{17} \mathrm{ClN}_{2} \mathrm{O}: \mathrm{C}, 61.78 ; \mathrm{H}, 6.78$, found: $\mathrm{C}, 62.02 ; \mathrm{H}, 6.76$.<smiles>CC(C)(C)NC(=O)CCc1ccccc1CCc1ccccc1CC(=O)NC(C)(C)C</smiles>

$N$-tert-Butyl-2-(2-\{2-[2-(tert-butylcarbamoyl-methyl)-phenyl]-ethyl $\}$-phenyl)-acetamide $\mathbf{1 6}$ was prepared according to Method 4 using a solution of the corresponding xanthate $(303 \mathrm{mg}$, $0.93 \mathrm{mmol})$ in octane $(9 \mathrm{~mL})$. Lauroyl peroxide $(290 \mathrm{mg}, 0.73 \mathrm{mmol}, 0.8 \mathrm{eq})$ was then added portionwise (20 mol \% per 20 minutes) to the refluxing solution. The crude mixture was then cooled to room temperature, concentrated under reduced pressure and purified by flash column chromatography (silica gel, petroleum ether-diethyl ether, 10:0 to 4:6) to give compound 16 in $\mathbf{5 5 \%}$ yield as white crystals. (m.p. $=148-151{ }^{\circ} \mathrm{C}$, recrystallized with heptane/ethyl acetate). ${ }^{1} \mathrm{H}$ NMR $\left(\mathrm{CDCl}_{3}, 400 \mathrm{MHz}\right) \delta 7.23$ (m, 8H, CHar.), 5.84 (bs, 2H), $3.53\left(\mathrm{~s}, 4 \mathrm{H}, \mathrm{CH}_{2}\right), 2.82\left(\mathrm{~s}, 4 \mathrm{H}, \mathrm{CH}_{2}\right), 1.30\left(\mathrm{~s}, 18 \mathrm{H}, \mathrm{CH}_{3}\right) \mathrm{ppm} ;{ }^{13} \mathrm{C} \mathrm{NMR}\left(\mathrm{CDCl}_{3}, 100 \mathrm{MHz}\right) \delta$ 170.6 (CO), 140.3 (Car.), 133.7 (Car.), 131.0 (CHar.), 130.3 (CHar.), 127.8 (CHar.), 127.0 (CHar.), $51.3(C), 41.9\left(\mathrm{CH}_{2}\right), 35.0\left(\mathrm{CH}_{2}\right), 28.8\left(\mathrm{CH}_{3}\right)$ ppm. IR $\left(\mathrm{CH}_{2} \mathrm{Cl}_{2}\right)$ 3289, 3063, 2963, 2927, 2870, 1659, 1540, 1511, 1491, 1451, 1391, 1362, 1262, 1225, $1193 \mathrm{~cm}^{-1} . \mathrm{MS}\left(\mathrm{CI}+\mathrm{NH}_{3}\right)$ $\mathrm{m} / \mathrm{z} 408\left(\mathrm{MH}^{+}\right)$. Anal. calcd. for $\mathrm{C}_{26} \mathrm{H}_{36} \mathrm{~N}_{2} \mathrm{O}_{2}: \mathrm{C}, 76.43 ; \mathrm{H}, 8.88$, found: $\mathrm{C}, 76.88 ; \mathrm{H}, 9.03$. 


\section{Preparation of the radical precursor $22 \mathrm{~b}$}

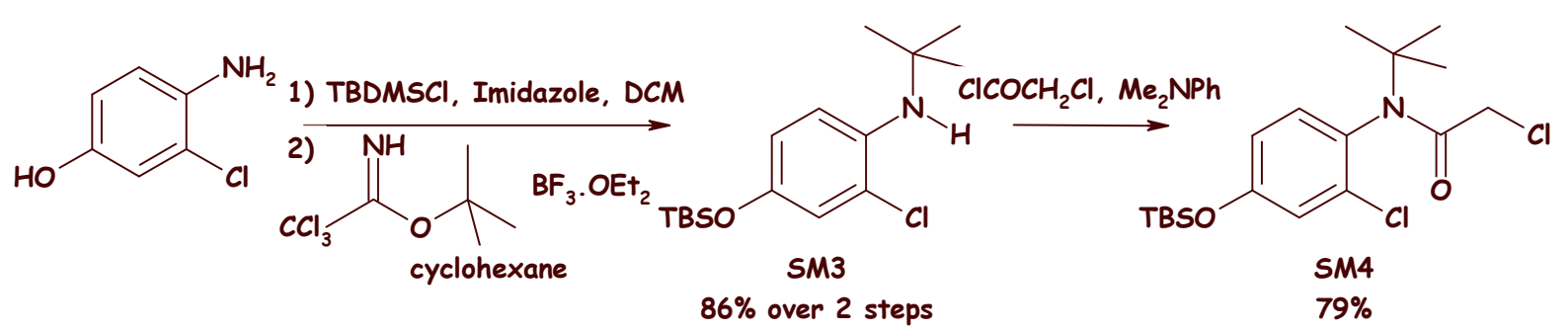

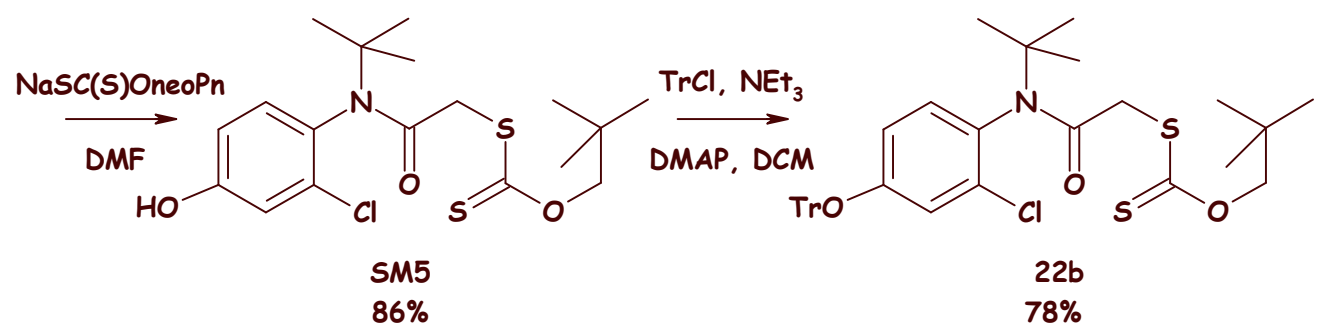

To a stirred solution of 4-amino-3-chlorophenol hydrochloride ( $1 \mathrm{~g}, 5.55 \mathrm{mmol})$ in dichloromethane $(10 \mathrm{~mL})$ at r.t. under argon, were successively added imidazole $(1.51 \mathrm{~g}, 22.2$ mmol, 4 eq) and tert-Butyldimethylsilyl chloride (1.260 g, $8.36 \mathrm{mmol}, 1.5 \mathrm{eq})$. The reaction being regularly monitored for the disappearance of the starting material by thin-layer chromatography, the starting material was totally consumed after 2 hours. The resulting mixture was diluted with water, extracted with dichloromethane, dried and concentrated under reduced pressure. The residue was used with no further purification in the next reaction.

A small amount was purified for characterization by flash column chromatography (silica gel, petroleum ether-diethyl ether, 10:0 to 9:1) to give 4-(tert-Butyl-dimethyl-silanyloxy)-2chloro-phenylamine as a yellow oil. ${ }^{1} \mathrm{H} \mathrm{NMR}\left(\mathrm{CDCl}_{3}, 400 \mathrm{MHz}\right) \delta 6.80(\mathrm{~d}, 1 \mathrm{H}, \mathrm{CHar}$., $\mathrm{J}=2.5 \mathrm{~Hz}$ ), 6.66 (d, 1H, CHar., J=8.6Hz), 6.60 (dd, 1H, CHar., J=2.6Hz, J=8.6Hz), 3.76 (bs, $\left.2 \mathrm{H}, \mathrm{NH}_{2}\right), 0.98\left(\mathrm{~s}, 9 \mathrm{H}, \mathrm{CH}_{3}\right), 0.17\left(\mathrm{~s}, 6 \mathrm{H}, \mathrm{CH}_{3}\right) \mathrm{ppm} ;{ }^{13} \mathrm{C} \mathrm{NMR}\left(\mathrm{CDCl}_{3}, 100 \mathrm{MHz}\right) \delta 148.1$ (Car.), 137.2 (Car.), 120.9 (CHar.), 119.7 (CHar.), 119.6 (Car.), 116.6 (CHar.), $25.8\left(\mathrm{CH}_{3}\right)$, 18.2 (C), -4.4 $\left(\mathrm{CH}_{3}\right) \mathrm{ppm}$. IR (neat) 3464, 3376, 2956, 2929, 2885, 2858, 1601, 1498, 1472 , $1302,1263,1214,944,839,801,780,685 \mathrm{~cm}^{-1}$. MS (CI+NH 3$) \mathrm{m} / \mathrm{z} 258,260\left(\mathrm{MH}^{+}\right)$.

To a stirred solution of the resulting residue in cyclohexane $(10 \mathrm{~mL})$ at r.t. under argon, were successively added tert-Butyl 2,2,2-trichloroacetimidate (2.5 mL, $13.9 \mathrm{mmol}, 2.5 \mathrm{eq})$ and 3 drops of $\mathrm{BF}_{3} \cdot \mathrm{OEt}_{2}$. The formation of a white precipitate was observed. The reaction was monitored by TLC and after 3 hours, tert-Butyl 2,2,2-trichloroacetimidate $(0.5 \mathrm{~mL}, 2.79$ mmol, $0.5 \mathrm{eq})$ and 3 drops of $\mathrm{BF}_{3} . \mathrm{OEt}_{2}$ were added. The reaction was stirred overnight and tert-Butyl 2,2,2-trichloroacetimidate $(1.1 \mathrm{~mL}, 6.14 \mathrm{mmol}, 1.1 \mathrm{eq})$ and 3 drops of $\mathrm{BF}_{3} . \mathrm{OEt}_{2}$ were added again. When starting material was totally consumed, the cyclohexane was evaporated in vacuo and the resulting crude residue was purified by flash column chromatography (silica gel, petroleum ether-diethyl ether with $1 \% \mathrm{NEt}_{3}, 10: 0$ to 98:2) to give compound SM3 in $\mathbf{8 6 \%}$ yield over 2 steps as a yellow oil. ${ }^{1} \mathrm{H}$ NMR $\left(\mathrm{CDCl}_{3}, 400 \mathrm{MHz}\right) \delta 6.92$ (d, 1H, CHar., J=8.8Hz), 6.84 (d, 1H, CHar., J=2.8Hz), 6.64 (dd, 1H, CHar., J=2.8Hz, $\mathrm{J}=8.8 \mathrm{~Hz}), 3.83$ (bs, $1 \mathrm{H}, \mathrm{NH}), 1.32\left(\mathrm{~s}, 9 \mathrm{H}, \mathrm{CH}_{3}\right), 0.98\left(\mathrm{~s}, 9 \mathrm{H}, \mathrm{CH}_{3}\right), 0.18\left(\mathrm{~s}, 6 \mathrm{H}, \mathrm{CH}_{3}\right) \mathrm{ppm}$; ${ }^{13} \mathrm{C}$ NMR $\left(\mathrm{CDCl}_{3}, 100 \mathrm{MHz}\right) \delta 148.3$ (Car.), 137.5 (Car.), 123.7 (Car.), 121.0 (CHar.), 119.4 (CHar.), 118.9 (CHar.), $52.5(C), 30.2\left(\mathrm{CH}_{3}\right), 25.8\left(\mathrm{CH}_{3}\right), 18.2(C),-4.4\left(\mathrm{CH}_{3}\right) \mathrm{ppm} . \mathrm{IR}$ (neat) $3417,2959,2895,2858,1604,1507,1472,1391,1364,1283,1255,1226,1040,948,838$, $781,685 \mathrm{~cm}^{-1}$. MS $\left(\mathrm{CI}+\mathrm{NH}_{3}\right) \mathrm{m} / \mathrm{z} 314,316\left(\mathrm{MH}^{+}\right)$. 
To a stirred solution of compound SM3 $(1.522 \mathrm{~g}, 4.86 \mathrm{mmol})$ in dichloromethane $(40 \mathrm{~mL})$ at r.t. under argon, were successively added $N, N$-dimethylaniline $(0.98 \mathrm{~mL}, 7.73 \mathrm{mmol}, 1.6$ eq) and chloroacetyl chloride $(0.5 \mathrm{~mL}, 6.28 \mathrm{mmol}, 1.3 \mathrm{eq})$. The solution was stirred at r.t. until starting material was totally consumed. The crude mixture was poured into saturated aqueous $\mathrm{Na}_{2} \mathrm{CO}_{3}$, extracted with dichloromethane, dried and concentrated under reduced pressure. The resulting crude residue was purified by flash column chromatography (silica gel, petroleum ether-diethyl ether with $1 \% \mathrm{NEt}_{3}, 10: 0$ to $99: 1$ ) to give compound SM4 in 79\% yield as a colorless oil. ${ }^{1} \mathrm{H}$ NMR $\left(\mathrm{CDCl}_{3}, 400 \mathrm{MHz}\right) \delta 7.07(\mathrm{~d}, 1 \mathrm{H}, \mathrm{CHar}$., J=8.6Hz), 6.95 (d, 1H, CHar., J=2.7Hz), 6.76 (dd, 1H, CHar., J=2.7Hz, J=8.6Hz), 3.69 (d, 1H, $\mathrm{CH}_{2}$, $\mathrm{J}=14.1 \mathrm{~Hz}), 3.63\left(\mathrm{~d}, 1 \mathrm{H}, \mathrm{CH}_{2}, \mathrm{~J}=14.1 \mathrm{~Hz}\right), 1.39$ (s, 9H, $\left.\mathrm{CH}_{3}\right), 0.97\left(\mathrm{~s}, 9 \mathrm{H}, \mathrm{CH}_{3}\right), 0.23(\mathrm{~s}, 6 \mathrm{H}$, $\left.\mathrm{CH}_{3}\right) \mathrm{ppm} ;{ }^{13} \mathrm{C} \mathrm{NMR}\left(\mathrm{CDCl}_{3}, 100 \mathrm{MHz}\right) \delta 165.6$ (CO), 156.6 (Car.), 134.9 (Car.), 131.9 (CHar.), 131.6 (Car.), 121.8 (CHar.), 119.4 (CHar.), $60.3(C), 44.8\left(\mathrm{CH}_{2}\right), 28.5\left(\mathrm{CH}_{3}\right), 25.5$ $\left(\mathrm{CH}_{3}\right), 18.2(\mathrm{C}),-4.4\left(\mathrm{CH}_{3}\right) \mathrm{ppm}$. IR (neat) 2957, 2951, 2858, 1682, 1598, 1523, 1494, 1394, $1362,1283,1256,1211,1193,946,833,783,685 \mathrm{~cm}^{-1} . \mathrm{MS}\left(\mathrm{CI}+\mathrm{NH}_{3}\right) \mathrm{m} / \mathrm{z} 389,391,393$ $\left(\mathrm{MH}^{+}\right)$.

To a solution of compound SM4 (1.464 g, $3.75 \mathrm{mmol})$ in dimethylformamide $(8 \mathrm{~mL})$ under argon was added Sodium $O$-Neopentyl xanthate ${ }^{7}(839 \mathrm{mg}, 4.51 \mathrm{mmol}, 1.2 \mathrm{eq})$ and the reaction mixture was heated at $50{ }^{\circ} \mathrm{C}$ for 3 hours. After consumption of all the starting material, the resulting mixture was diluted with water, extracted with ether, washed several times with brine, dried and concentrated. The residue was purified by flash column chromatography (silica gel, petroleum ether-diethyl ether, 10:0 to 7:3) to give compound SM5 in $\mathbf{8 6 \%}$ yield as white crystals. (m.p. $=188-191^{\circ} \mathrm{C}$, recrystallized with heptane/ethyl acetate). ${ }^{1} \mathrm{H} \mathrm{NMR}\left(\mathrm{CDCl}_{3}, 400 \mathrm{MHz}\right) \delta 7.11(\mathrm{~d}, 1 \mathrm{H}, \mathrm{CHar} ., \mathrm{J}=8.6 \mathrm{~Hz}), 6.96(\mathrm{~d}, 1 \mathrm{H}, \mathrm{CHar} ., \mathrm{J}=2.7 \mathrm{~Hz})$, 6.78 (dd, 1H, CHar., J=2.7Hz, J=8.6Hz), 4.13 (s, 2H, CH2), $3.72\left(\mathrm{~d}, 1 \mathrm{H}, \mathrm{CH}_{2}, \mathrm{~J}=16.2 \mathrm{~Hz}\right), 3.59$ $\left(\mathrm{d}, 1 \mathrm{H}, \mathrm{CH}_{2}, \mathrm{~J}=16.2 \mathrm{~Hz}\right), 1.38\left(\mathrm{~s}, 9 \mathrm{H}, \mathrm{CH}_{3}\right), 0.91\left(\mathrm{~s}, 9 \mathrm{H}, \mathrm{CH}_{3}\right) \mathrm{ppm} ;{ }^{13} \mathrm{C} \mathrm{NMR}\left(\mathrm{CDCl}_{3}, 100\right.$ MHz) $\delta 214.1$ (CS), 166.8 (CO), 158.0 (Car.), 134.7 (Car.), 131.9 (CHar.), 130.7 (Car.), 117.2 (CHar.), 115.1 (CHar.), $83.5\left(\mathrm{CH}_{2}\right), 60.3(\mathrm{C}), 41.9\left(\mathrm{CH}_{2}\right), 31.8(\mathrm{C}), 28.6\left(\mathrm{CH}_{3}\right), 26.5\left(\mathrm{CH}_{3}\right)$ ppm. IR $\left(\mathrm{CH}_{2} \mathrm{Cl}_{2}\right) 3567,3304,2965,1658,1606,1495,1394,1366,1273,1250,1232,1189$, $1070,952 \mathrm{~cm}^{-1}$. MS $\left(\mathrm{CI}+\mathrm{NH}_{3}\right) \mathrm{m} / \mathrm{z} 403\left(\mathrm{MH}^{+}\right)$. Anal. calcd. for $\mathrm{C}_{18} \mathrm{H}_{26} \mathrm{ClNO}_{3} \mathrm{~S}_{2}: \mathrm{C}, 53.52 ; \mathrm{H}$, 6.49, found: $\mathrm{C}, 53.85 ; \mathrm{H}, 6.32$.

To a stirred solution of compound SM5 $(1.315 \mathrm{~g}, 3.26 \mathrm{mmol})$ in dichloromethane $(70 \mathrm{~mL})$ at r.t. under argon, were successively added triethylamine $(1.14 \mathrm{~mL}, 8.18 \mathrm{mmol}, 2.5 \mathrm{eq})$, 4dimethylaminopyridine $(74 \mathrm{mg}, 0.60 \mathrm{mmol}, 0.2 \mathrm{eq})$ and trityl chloride $(1.135 \mathrm{~g}, 4.07 \mathrm{mmol}$, $1.2 \mathrm{eq})$. The reaction being regularly monitored for the disappearance of the starting material by thin-layer chromatography, the starting material was totally consumed after 3 hours. The resulting mixture was poured into saturated aqueous $\mathrm{NaHCO}_{3}$, extracted with dichloromethane, dried and concentrated under reduced pressure. The residue was purified by flash column chromatography (silica gel, petroleum ether-diethyl ether, 10:0 to 9:1) followed by fractional crystallization to give compound $\mathbf{2 2 b}$ in $\mathbf{7 8 \%}$ yield as white crystals. (m.p. $=$ $122-125^{\circ} \mathrm{C}$, recrystallized with heptane). ${ }^{1} \mathrm{H} \mathrm{NMR}\left(\mathrm{CDCl}_{3}, 400 \mathrm{MHz}\right) \delta 7.46(\mathrm{~m}, 6 \mathrm{H}, \mathrm{CHar}$.), 7.28 (m, 9H, CHar.), 6.91 (d, 1H, CHar., J=8.7Hz), 6.87 (d, 1H, CHar., J=2.7Hz), 6.64 (dd, $1 \mathrm{H}, \mathrm{CHar} ., \mathrm{J}=2.7 \mathrm{~Hz}, \mathrm{~J}=8.7 \mathrm{~Hz}), 4.22\left(\mathrm{~d}, 1 \mathrm{H}, \mathrm{OCH}_{2}, \mathrm{~J}=10.5 \mathrm{~Hz}\right), 4.19\left(\mathrm{~d}, 1 \mathrm{H}, \mathrm{OCH}_{2}, \mathrm{~J}=10.5 \mathrm{~Hz}\right.$ ), $3.51\left(\mathrm{~d}, 1 \mathrm{H}, \mathrm{CH}_{2}, \mathrm{~J}=16.3 \mathrm{~Hz}\right), 3.43\left(\mathrm{~d}, 1 \mathrm{H}, \mathrm{CH}_{2}, \mathrm{~J}=16.3 \mathrm{~Hz}\right), 1.30\left(\mathrm{~s}, 9 \mathrm{H}, \mathrm{CH}_{3}\right), 0.99(\mathrm{~s}, 9 \mathrm{H}$, $\left.\mathrm{CH}_{3}\right)$ ppm ; ${ }^{13} \mathrm{C} \mathrm{NMR}\left(\mathrm{CDCl}_{3}, 100 \mathrm{MHz}\right) \delta 214.3$ (CS), 165.9 (CO), 157.1 (Car.), 146.9 (Car.), 143.4 (Car.), 133.9 (Car.), 133.0 (Car.), 130.7 (CHar.), 128.9 (CHar.), 128.0 (CHar.), 127.9 (CHar.), 127.9 (CHar.), 127.6 (CHar.), 127.3 (CHar.), 123.8 (CHar.), 121.4 (CHar.), $92.5(C), 83.5\left(\mathrm{OCH}_{2}\right), 60.1(\mathrm{C}), 42.0\left(\mathrm{CH}_{2}\right), 31.8(\mathrm{C}), 28.6\left(\mathrm{CH}_{3}\right), 26.6\left(\mathrm{CH}_{3}\right)$ ppm. IR

\footnotetext{
${ }^{7}$ See for example: Quiclet-Sire, B.; Zard, S. Z. J. Am. Chem. Soc. 1996, 118, 9190-9191.
} 
$\left(\mathrm{CH}_{2} \mathrm{Cl}_{2}\right) 3059,2961,1667,1596,1488,1448,1394,1366,1273,1230,1198,1070,1030$, 964, 910, 733, $702 \mathrm{~cm}^{-1}$. MS $\left(\mathrm{CI}+\mathrm{NH}_{3}\right) \mathrm{m} / \mathrm{z}$ 648, $650\left(\mathrm{MH}^{+}\right)$.<smiles>CC(C)(C)N1C(=O)CC12C=CC(=O)C=C2Cl</smiles>

1-tert-Butyl-5-chloro-1-aza-spiro[3.5]nona-5,8-diene-2,7-dione 30 was prepared according to Method 4 using a solution of the corresponding xanthate $(485 \mathrm{mg}, 0.75 \mathrm{mmol})$ 1,2dichloroethane $(7.5 \mathrm{~mL})$. Lauroyl peroxide $(389 \mathrm{mg}, 0.98 \mathrm{mmol}, 1.3 \mathrm{eq})$ was then added portionwise to the refluxing solution ( $60 \mathrm{~mol} \%$ per 90 minutes then $10 \mathrm{~mol} \%$ ). The crude mixture was then cooled to room temperature, concentrated under reduced pressure and purified by flash column chromatography (silica gel, petroleum ether-diethyl ether, 10:0 to $2: 8)$ to give compound $\mathbf{3 0}$ in $\mathbf{4 1 \%}$ yield as a yellow oil. ${ }^{1} \mathrm{H} \mathrm{NMR}\left(\mathrm{CDCl}_{3}, 400 \mathrm{MHz}\right) \delta 7.09$ (d, $1 \mathrm{H}, \mathrm{CH}, \mathrm{J}=10.0 \mathrm{~Hz}), 6.59$ (d, $1 \mathrm{H}, \mathrm{CH}, \mathrm{J}=1.8 \mathrm{~Hz}), 6.40$ (dd, $1 \mathrm{H}, \mathrm{CH}, \mathrm{J}=1.8 \mathrm{~Hz}, \mathrm{~J}=10.0 \mathrm{~Hz})$, $3.24\left(\mathrm{~d}, 1 \mathrm{H}, \mathrm{CH}_{2}, \mathrm{~J}=14.6 \mathrm{~Hz}\right), 2.92\left(\mathrm{~d}, 1 \mathrm{H}, \mathrm{CH}_{2}, \mathrm{~J}=14.6 \mathrm{~Hz}\right), 1.34\left(\mathrm{~s}, 9 \mathrm{H}, \mathrm{CH}_{3}\right) \mathrm{ppm} ;{ }^{13} \mathrm{C} \mathrm{NMR}$ $\left(\mathrm{CDCl}_{3}, 100 \mathrm{MHz}\right) \delta 183.3(\mathrm{CO}), 163.9(\mathrm{NCO}), 155.9(\mathrm{C}), 148.8(\mathrm{CH}), 130.8(\mathrm{CH}), 129.8$ $(\mathrm{CH}), 58.5(\mathrm{C}), 57.0(\mathrm{C}), 47.4\left(\mathrm{CH}_{2}\right), 27.8\left(\mathrm{CH}_{3}\right) \mathrm{ppm}$. IR (neat) 3346, 2976, 2931, 1766, $1664,1599,1503,1369,1327,1286,1259,1226,1040,983,893,809 \mathrm{~cm}^{-1} . \mathrm{MS}\left(\mathrm{CI}+\mathrm{NH}_{3}\right)$ $\mathrm{m} / \mathrm{z} 240,242\left(\mathrm{MH}^{+}\right)$. 


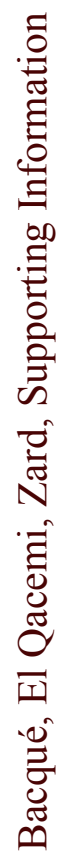

$\downarrow 乙 8 ' 乙$

$629^{\circ} \varepsilon$

19.54

- 4.09

○

$\stackrel{\circ}{i}$

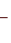

6टง

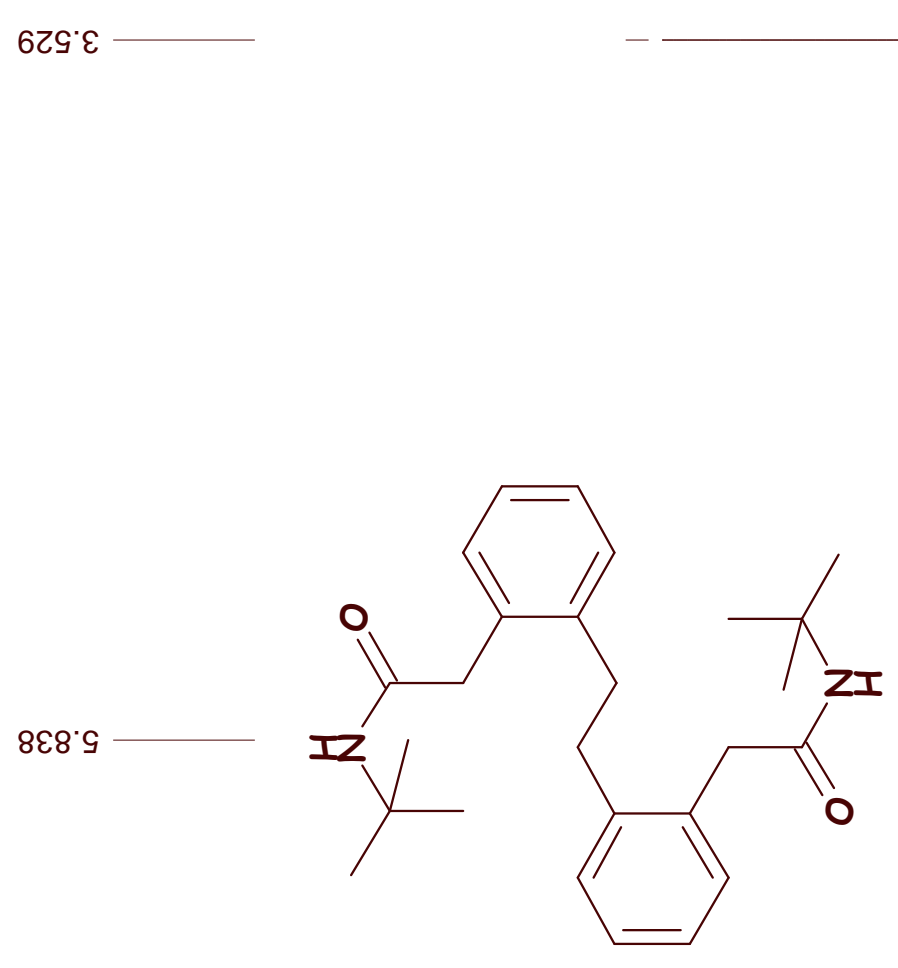

8६ट $\angle$

ट Z' $\angle$

StZ' $L$

$0 \angle Z^{\circ} \angle$

] 4.00

$\frac{\infty}{\infty}$

$\frac{E}{E}$ 


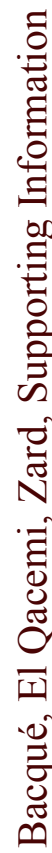

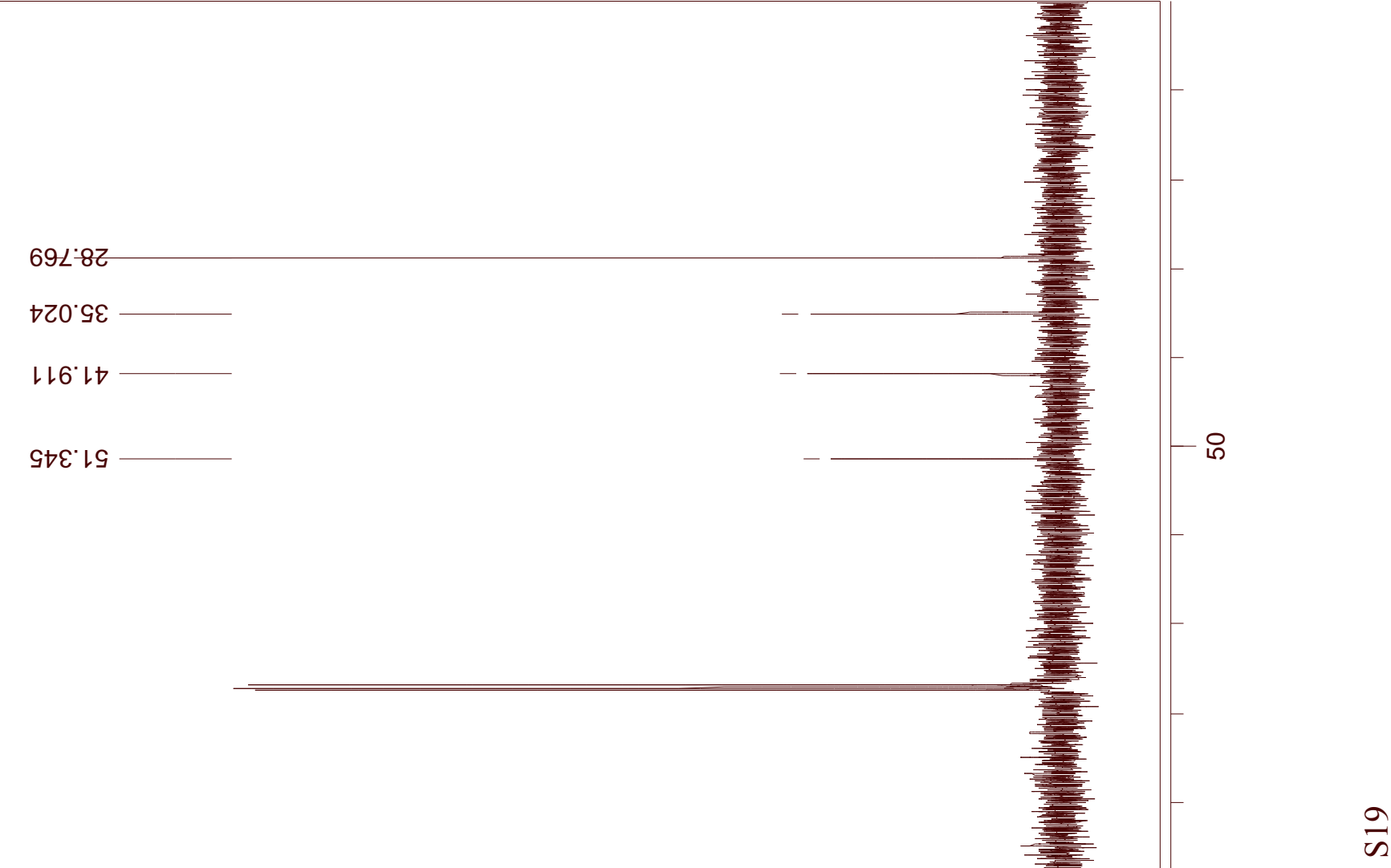

996.92

††8' $\angle 2 L$

$\varepsilon \subseteq \varepsilon \cdot 0 \varepsilon$ เ

S00 $L E$ L

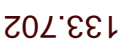

टLE'0tト

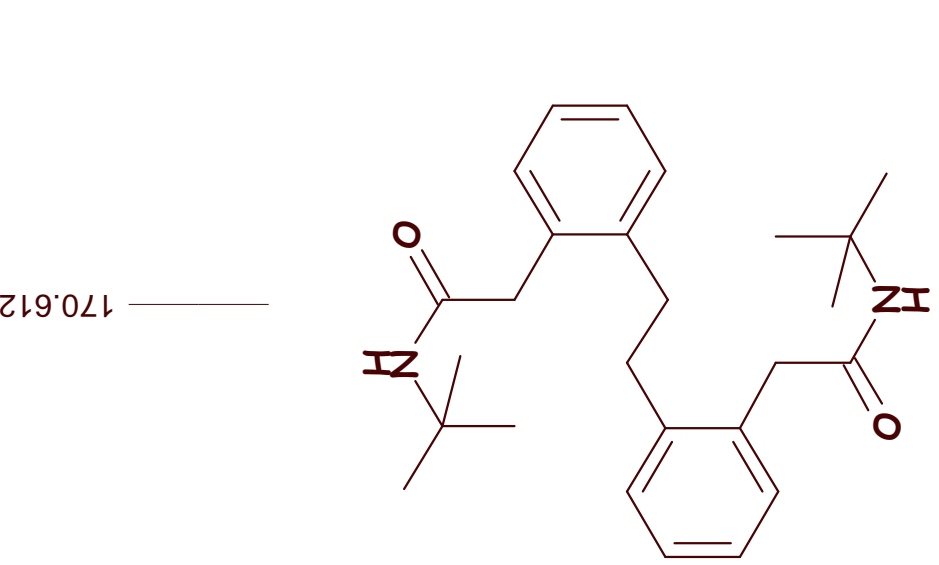




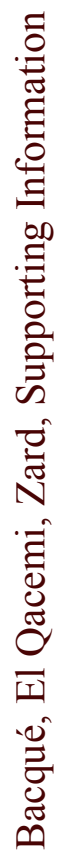

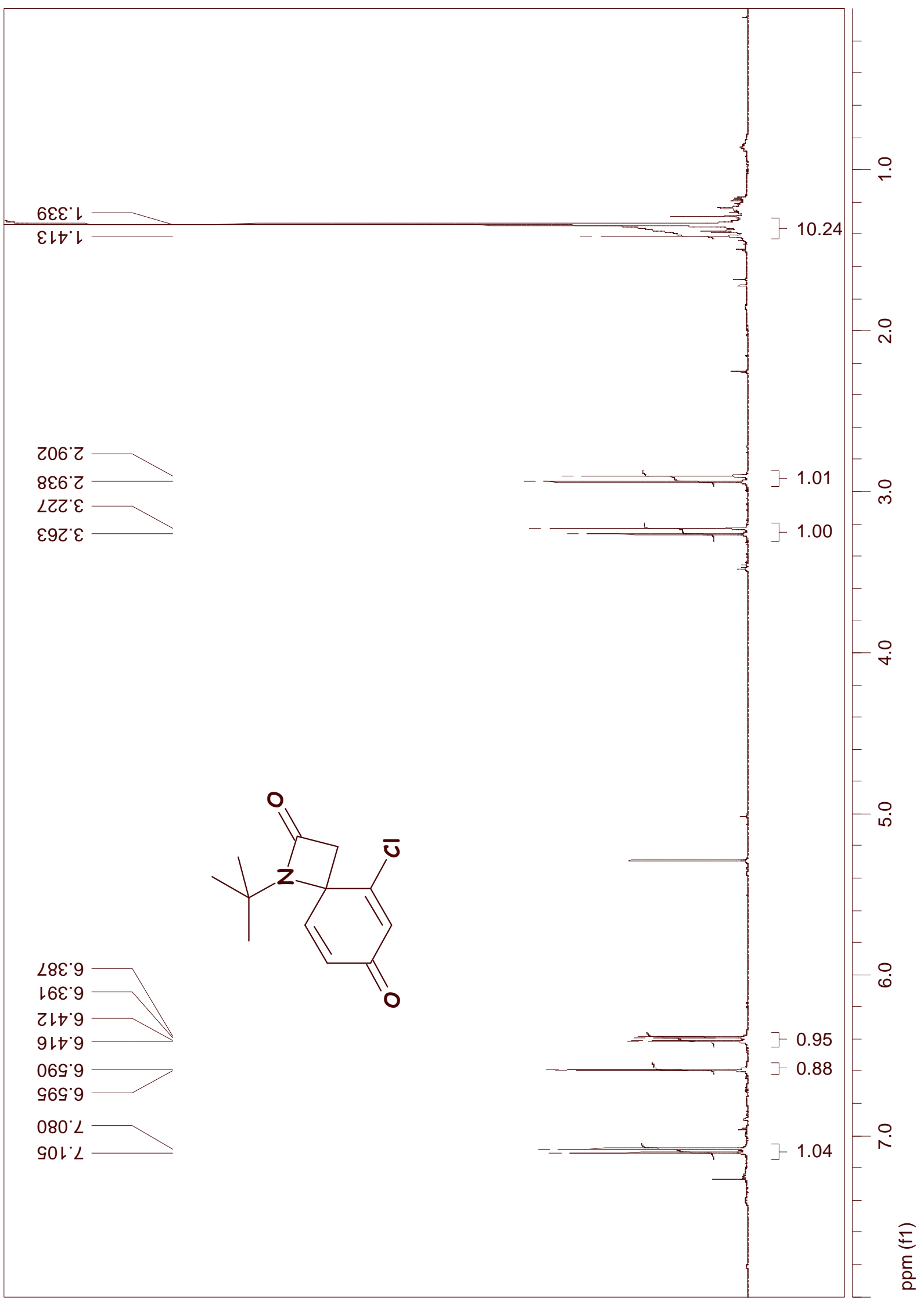




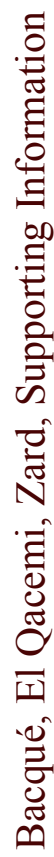

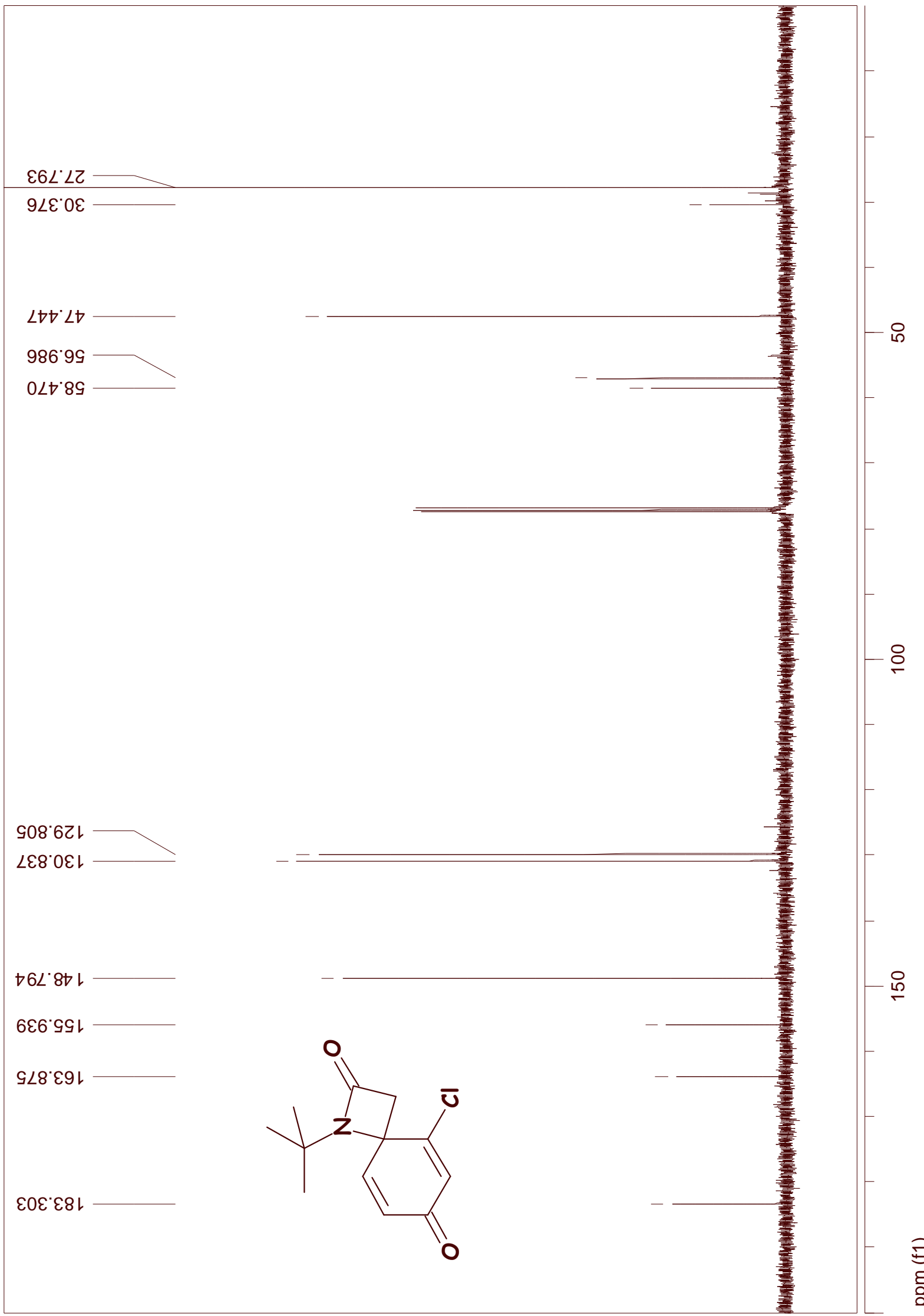

